\title{
The Hotelling-Downs Model with Runoff Voting
}

\author{
Sandro Brusco* $\quad$ Marcin Dziubiński ${ }^{\dagger} \quad$ Jaideep Roy ${ }^{\ddagger}$
}

September 2010

\begin{abstract}
We consider the Hotelling-Downs model with $n \geq 2$ office seeking candidates and runoff voting. We show that Nash equilibria in pure strategies always exist and that there are typically multiple equilibria, both convergent (all candidates are located at the median) and divergent (candidates locate at distinct positions), though only divergent equilibria are robust to free entry. Moreover, two-policy equilibria exist under any distribution of voters' ideal policies, while equilibria with more than two policies exist generically but under restrictive conditions that we characterize. In this sense, our analysis suggests that two-policy equilibria are the most prominent outcomes.
\end{abstract}

Keywords: Downs, Free Entry, Runoff System, Equilibrium

JEL: D01, D03, D70

\section{Introduction}

The Hotelling-Downs (HD henceforth; see Hotelling (1929) and Downs (1957)) model has remained central in the theoretical literature on electoral competition. In the classic version of that model, two purely office seeking politicians choose their policy platforms independently and simultaneously and voters vote to elect their leader based upon their preferences over the policy space. The model supports the celebrated result that a unique Nash equilibrium exists for any distribution of voters' ideal policies where both players choose the platform of the median voter. Osborne (1993) however has shown that in the HD model with sincere voting ${ }^{1}$ under the first-past-the-post (FPTP) system, if there are more than two players, a Nash equilibrium in pure strategies fails to exist generically. While the FPTP system is widely used and by

\footnotetext{
* Department of Economics and Center for Game Theory, Stony Brook University, NY 11794-4384, USA, Tel.: +1631632 7548; Email: sandro.brusco@stonybrook.edu

${ }^{\dagger}$ Institute of Informatics, Faculty of Mathematics, Informatics and Mechanics, Warsaw University, Banacha 2, 02-097 Warsaw, Poland. Tel.: +48-22-5544503; Email: m.dziubinski@mimuw.edu.pl.

${ }^{\ddagger}$ Department of Economics, University of Birmingham, Edgbaston, Birmingham B15 2TT, UK, Tel.: +44 18952 65539; Email: j.roy.1@bham.ac.uk.

${ }^{1}$ Osborne (1993) assumes a continuum of voters.
} 
now well understood analytically, the Runoff system is no less popular, yet little studied. It is used in the French presidential, legislative and cantonal elections. It is also used to elect the presidents of many other European and Latin American nations including Austria, Bulgaria, Croatia, Cyprus, Finland, Poland, Portugal, Romania, Slovakia, Brazil, Chile and Columbia, to name a few.

In this paper we investigate the existence and characterisation of Nash equilibria in the HD model when the voting system is runoff, rather than FPTP. A runoff system can be generally described as follows. In the first round a candidate is elected if she gets the largest share of the votes and the share is at least $z \%$ of the votes ${ }^{2}$. If no candidate gets more than $z \%$ of the votes in the first round, then a subset of candidates is selected according to a pre-specified criterion to run in a second round, where the winner is decided by FPTP. The most widely used version has $z=50$, i.e. a candidate wins at the first round only if she gets the absolute majority; otherwise the subset of candidates moving to the second round is given by the two top voted candidates $^{3}$. Examples of the 'classic' version with $z=50$ and the two top vote-getters moving to the second round are given by the presidential elections in Chile, Colombia, France and Poland, as well as federal and statelevel offices in some US states. The presidential elections in Argentina and Costarica are examples of runoff systems with $z<50(z=45$ in Argentina and $z=40$ in Costarica).

We assume that at least three players are present; this is the only interesting case, since with two players, runoff is equivalent to FPTP. We show that for $n=3$ there is only one equilibrium which always exists, with all players located at the median. When $n>3$ however the equilibrium set expands considerably. Besides the convergent equilibria which exist for any $n \geq 3$ and for any distribution of voters' ideologies, there is a continuum of divergent equilibria in which the candidates choose positions different from the median. The intuition comes from the fact that in a runoff system one has to worry not only about the share of the vote in the first round, but also about what happens in the second round. To see that convergent equilibria where all players locate at the median always exist, consider a possible deviation. By moving slightly right or left a candidate can get (almost) $50 \%$ of the vote and thus be sure to be selected for the second round. However, in the second round the deviating candidate will face an opponent located

\footnotetext{
${ }^{2}$ There are some possible complications in actually used runoff systems that we are going to ignore in this paper. For example, in the French legislative elections winning at the first round requires not only getting at least $50 \%$ of the valid votes, but also at least $25 \%$ of registered voters. In this paper we will ignore abstention, so there is no difference between potential voters and actual voters. See Grofman (2008) for a general taxonomy of runoff methods.

${ }^{3} \mathrm{An}$ example with a different rule is given by the French legislative elections, where every candidate who gets at least $12.5 \%$ of the vote is allowed to run in the second round.
} 
at the median, meaning defeat with probability one. Thus, such deviation is unprofitable. To see how divergent equilibria can be sustained, consider four candidates and suppose that candidates 1 and 2 locate at $m-e$ while candidates 3 and 4 locate at $m+e$, where $m$ is the median and $e$ is sufficiently small. Each candidate collects one fourth of the votes and has an equal chance of being selected for the second round, where (whatever the consequent selection is) the two candidates will have an equal chance to win. Now consider a potential deviation by player 1. For $e$ small, moving slightly to the left will typically increase the vote share of the candidate, thus ensuring that player 1 will go to the second stage with probability 1 . However, at that stage the candidate will lose for sure, given that the position he occupies is the farthest away from the median. This makes the deviation unprofitable. Moving to the right, for $e$ small, cannot be profitable either, since candidate 1 ends up decreasing his vote share and being excluded from the second round.

In this intuitive example, multiple candidates occupy each of the extreme positions. We show that this is a general characteristic of divergent equilibria: extreme policies are always proposed by at least two players. We also show that in equilibrium all players who contest the election must have the same vote share in round one and that while in all equilibria any of the positions represented can win the election, positions closer to the median have a higher probability of winning. Thus, in a divergent equilibrium the probability of winning the elections is strictly decreasing with the distance from the median.

How many different policy platforms can be represented in a divergent equilibrium? We show that, for $n>3$, and for any arbitrary distribution of voters' ideologies, a continuum of two-policy equilibria exist. In each such equilibrium, the policy positions which are occupied are symmetric around the median and an equal number of candidates enter at each position. Next, we show that three-policy equilibria are generically possible, though unlike the two-policy case, existence is not always guaranteed. In every generic case, the middle policy is proposed by a single player while the extreme policies are symmetric about the median and are proposed by an equal number of players. Interestingly, this middle policy is never the median voter's ideal policy in any generic situation. It is important to note that in the FPTP system as studied in Osborne (1993), while no equilibrium exists generically, two-policy equilibria can exist while three policy equilibria must have each of the policies proposed by two candidates. ${ }^{4}$

We then address equilibria with more than three policy platforms. We show that such equilibria exist generically only if $n \geq 6$, although existence is not guaranteed and requires additional conditions on the distribution.

\footnotetext{
${ }^{4}$ The result about three policy equilibria is not shown in Osborne (1993). In our analysis we provide an argument that implies this result.
} 
Moreover, the observation made above regarding the internal policy in a 3 -policy equilibrium is a general feature: in any generic situation, all internal policies are proposed by exactly one player. Interestingly, extreme policies do not necessarily have to be symmetric around the median: in fact, if the number of policies is even, then these extreme policies cannot be symmetric, while if they are odd, equilibria can support symmetry as well as non-symmetry. Our analysis also suggests that although $k \geq 3$-policy equilibria exist generically, the conditions required on the distribution of ideal policies become very restrictive, and hence one may suggest they are less likely in an arbitrary election. If free entry is allowed for, it then turns out that the 2-policy equilibria are the most prominent outcomes.

Finally, we consider how the results on the equilibrium set vary when variations on the classic rules are adopted; specifically, we consider the case in which $z<50$ and the case in which more than two candidates are admitted to the second round. We show that in these cases an equilibrium may fail to exist, and that when equilibria exist, they are necessarily divergent and that they tend to be more 'extremist' (the candidates locate farther away from the median) than in the classic case.

The rest of the paper is structured as follows. In Section 2 we discuss the literature on the subject. Section 3 spells out the model. In Section 4 we characterize the equilibrium set and in Section 5 we discuss under what conditions various types of equilibria exist. Section 6 is dedicated to the analysis of equilibria in which more than two policies are proposed and Section 7 explores variations on the 'classic' case. Section 8 contains the conclusions. All proofs are collected in the Appendix.

\section{Related literature}

The two papers most closely related to our work are Haan and Volkerink (2001) and Callander (2005). The first paper studies exactly the HD model with runoff (case $z=50$ ) to address convergent equilibria and shows that for all distributions of voters' ideologies, an equilibrium always exists where all players enter as candidates at the median. We show that this is only a subset of the equilibrium set, and that when there are at least 4 players there are many divergent equilibria, i.e. equilibria in which the candidates locate at multiple policy positions. Furthermore, we show that for some variations of the 'classic' rule the convergent equilibrium disappears ${ }^{5}$. More importantly, it also disappears if there is free entry of players in the political system, while divergent equilibria are in this sense robust.

\footnotetext{
${ }^{5}$ Matsushima (2007) extends the HD model to allow for aggregate uncertainty (i.e. the position of the median voter is uncertain at the time players choose their policy platforms). He shows that when the distribution function for the position of the median voter is continuous, the single-policy convergence result of Haan and Volkerink (2001) fails to hold.
} 
Callander (2005) considers a model which is slightly different from ours. The variation is that he considers a two-period entry game in which $n$ established candidates select a location in period 1 and then a potential entrant decides whether to enter, and at what location, in period 2 (this is an extension of the Palfrey (1984) model of entry deterrence $\left.{ }^{6}\right)$. The focus of the paper is on two-candidate equilibria under runoff voting, since the existence of such equilibria challenges the conjecture (due to Duverger) that the runoff system typically supports many more candidates ${ }^{7}$. Differently from this paper, Callander (2005) does not try to characterize the whole equilibrium set; rather, he proposes some equilibria, without discussing the general properties that equilibrium configurations have to satisfy. In this paper we instead start discussing the properties that any equilibrium must satisfy and then proceed to characterize the whole set of generic equilibria.

One important assumption made by Callander (2005) is that the entrant is less recognizable than the other candidates, so that if she enters at a position already occupied by an incumbent she receives no votes. He shows that when there are two incumbents (besides the potential entrant) then all the equilibria are divergent. In our model, the only equilibrium when there are less than 4 players is convergent and we do not have equilibria in which only two candidates enter; Callander obtains such an equilibrium because of his assumption on the advantage of incumbency ${ }^{8}$. Another important difference with Callander (2005) is that he considers only distributions with a density symmetric around the median and increasing up to the median (i.e. 'bell-shaped'), a set of distributions which is non-generic. We consider all non-atomic distributions and show that the multi-policy equilibria discussed in Callander (2005) can be obtained generically though not universally, while two-policy equilibria are robust in this sense.

A number of papers have analysed the runoff rule in models different from the classic HD model, usually comparing FPTP and runoff. Osborne and Slivinski (1996) show that in a citizen-candidate model multi-candidate equilibria are more likely under runoff than under FPTP. Also, in every twocandidate equilibrium, runoff reduces extremism when compared to FPTP. Bordignon and Tabellini (2009) compare FPTP versus runoff voting in a model where four politicians can form pre-electoral coalitions and present their policy platforms, while the voters are of four ideological types and voting is sincere. They show that under runoff the number of candidates

\footnotetext{
${ }^{6}$ In Palfrey (1984), there are exactly two established candidates and they do not have the option to quit the competition. In Callander (2005) this restriction is relaxed.

${ }^{7}$ Wright and Riker (1989) present empirical evidence suggesting that the runoff systems tend to have a larger number of candidates. See also Cox (1997) for an analytical study of the Duverger's Hypothesis under the runoff rule.

${ }^{8}$ The assumption that the entrant gets zero votes when its platform coincide with that of an incumbent is stronger than needed. Callander's results on two-candidate equilibria could be obtained simply by assuming that an entrant gets fewer votes than an incumbent located at the same policy position.
} 
is typically larger than under FPTP, although the influence of extremist voters is smaller as their bargaining power is reduced. Finally, Messner and Polborn (2007) study electoral competition with a small number of voters who are able to form coalitions and compare the two voting systems, taking the policies as exogenously given. They find that if there are three policies, then no strong equilibrium exists under the FPTP rule while it does so under runoff, provided there is a Condorcet winner. A coalition proof equilibrium appears in both systems even when there is a Condorcet cycle.

\section{The model}

We adopt notation and terminology from Osborne (1993). A policy is a point on the real line $\mathbb{R}$. Let $N=\{1, \ldots, n\}$ be the set of players, with $n \geq 3$. The strategy set for each player $i \in N$ is $S_{i}=\mathbb{R} \cup\{$ Out $\}$ and a pure strategy for player $i \in N$ is an element $s_{i} \in S_{i}$. If $s_{i} \in \mathbb{R}$, then it is the policy that player $i$ proposes in order to contest the elections as a candidate, while if $s_{i}=$ Out, then the player chooses to stay out of competition. A strategy profile is denoted by $s \in \prod_{i \in N} S_{i}$. Players act simultaneously and independently.

There is a continuum of voters, each having a unique ideal policy in $\mathbb{R}$. Ideal policies of the voters are distributed over $\mathbb{R}$ according to a distribution $F$. We will consider the class of distributions that can be represented by a density $f$ with a connected support, and we will call this class $\mathcal{F}$. If $f \in \mathcal{F}$ then there is a unique median, which will be denoted by $m$. We will also refer to the quantiles of the distribution $F$, denoting the $k^{\prime}$ 'th $p$-quantile (i.e. the point $x \in \mathbb{R}$ such that $F(x)=\frac{k}{p}$ ) by $q_{k / p}$. When $f \in \mathcal{F}$ the quantiles are uniquely determined for all strictly positive integers $k$ and $p$ with $k<p$.

Voting is sincere, that is each voter votes for a candidate offering a policy that is closest to his ideal point. Given a policy $x \in \mathbb{R}$ the mass of voters that vote for that policy is called the constituency of $x$. Left and right constituencies are defined in the usual way. The left constituency of a policy $x$ will be denoted by $\sigma_{1}(x)$, the right constituency of $x$ will be denoted by $\sigma_{\mathrm{r}}(x)$ and the total constituency of $x$ will be denoted by $\sigma(x)$. If left and right constituencies are equal, then the support of the policy will be called balanced. If $k$ candidates offer the same policy, then each of them receives votes equal to the same fraction $1 / k$ of the constituency of that policy. Elections follow the runoff rule where the winner is determined as follows: if some candidate obtains strictly more than $z \%$ of votes, then she is the winner. Otherwise, the winner is the candidate who obtains a majority in the second round between the two candidates who obtained the most votes in the first round. All ties (be it in the selection of the two candidates from the first round, or the eventual winner from the second round) are broken with equiprobable draws. Except for Section 7, we will consider the 'classic' 
case of $z=50$.

Candidates are purely office-seeking, that is, they care only about winning the elections. More exactly, let $P_{i}(s)$ be the probability that player $i \in N$ is the elected candidate under strategy profile $s$. We assume that $s$ is strictly preferred to $s^{\prime}$ by candidate $i$ when either $P_{i}(s)>P_{i}\left(s^{\prime}\right)$ or $P_{i}(s)=P_{i}\left(s^{\prime}\right)=0$ and $s_{i}=$ Out, $s_{i}^{\prime} \neq$ Out. In other words, a candidate prefers strategy profiles where the probability of victory is higher and it prefers to stay out rather than running when the probability of victory is zero. We omit the natural assumption of preferring to win outright in the first round, because it does not affect the results obtained.

\subsection{Policy profile and genericity}

A policy profile is a collection $\mathcal{X}=(X, \varrho)$, where $X=\left\{x_{1}, \ldots, x_{k}\right\} \subseteq \mathbb{R}$ is called the set of policies and $\varrho: X \rightarrow \mathbb{N}_{+}$is a function that assigns to each policy $x_{i} \in X$ the number of candidates proposing $x_{i}$, with $\varrho\left(x_{i}\right) \geq 1$ for each $x_{i} \in X$. In particular, we will call a policy proposed by exactly one candidate an unary policy, a policy proposed by exactly two candidates a binary policy, etc. We will also use $\varrho(\mathcal{X})=\sum_{x_{i} \in X} \varrho(x)$ to denote the total number of candidates in the policy profile $\mathcal{X}=(X, \varrho)$. The policy profile $\mathcal{X}=(\varnothing, \varnothing)$ is called the empty policy profile. If $\mathcal{X}$ is non-empty and $X=\left\{x_{1}, \ldots, x_{k}\right\}$, then we will use a convention that $i<j$ implies $x_{i}<x_{j}$. Moreover, if $k>1$, then we will call such a profile divergent, and if $k=1$, then the profile will be called convergent.

Given a non-empty policy profile $\mathcal{X}=(X, \varrho)$ we will use

$$
b_{i}^{\mathcal{X}}=\frac{x_{i}+x_{i+1}}{2},
$$

(for $i \in\{1, \ldots, k-1\}$ ) to denote the border between the support of the policies $x_{i}$ and $x_{i+1}$. The border of any two policies identifies the position of voters indifferent between these policies.

Each strategy profile $s$ determines a policy profile $\mathcal{X}_{s}=\left(X_{s}, \varrho_{s}\right)$. If $s$ is a Nash equilibrium then $\mathcal{X}_{s}$ is called an equilibrium strategy profile.

A policy profile $\mathcal{X}=(X, \varrho)$ is called even if for any $x_{i} \in X$,

$$
\sigma\left(x_{i}\right)=\frac{\varrho\left(x_{i}\right)}{\varrho(\mathcal{X})}
$$

Under an even policy profile, each candidate obtains the same mass of votes, equal to $\frac{1}{\varrho(\mathcal{X})}$. As we will show, any equilibrium policy profile is even. Notice that in even policy profiles, the borders between supports of policies are proper $\varrho(\mathcal{X})$-quantiles. More precisely

$$
b_{i}^{\mathcal{X}}=\frac{q_{\sum_{j=1}^{i} \varrho\left(x_{j}\right)}}{\varrho(\mathcal{X})},
$$


that is it is the $\sum_{j=1}^{i} \varrho\left(x_{j}\right)^{\prime}$ th $\varrho(\mathcal{X})$-quantile.

We will focus on equilibria which are generic. More precisely, we consider the following notion of genericity. Let the distance between two density functions $f$ and $g$ in $\mathcal{F}$ be given by

$$
\|f-g\|=\int|f(x)-g(x)| d x
$$

and let

$$
B_{\delta}(f)=\{g \in \mathcal{F}\|\mid f-g\|<\delta\}
$$

be an open $\delta$-ball around $f$. Suppose that for a given distribution $f$ there is a Nash equilibrium $s_{f}$ inducing a policy profile $\mathcal{X}=(X, \varrho)$ with $X=$ $\left\{x_{1}, \ldots, x_{k}\right\}$. Then we say that the Nash equilibrium $s_{f}$ is generic if for each $\varepsilon>0$ it is possible to find $\delta>0$ such that for each $f^{\prime} \in B_{\delta}(f)$ there is a Nash equilibrium $s_{f^{\prime}}$ inducing a policy profile $\mathcal{X}^{\prime}=\left(X^{\prime}, \varrho\right)$ with $X^{\prime}=$ $\left\{x_{1}^{\prime}, \ldots, x_{k}^{\prime}\right\}$ such that $\left|x_{i}-x_{i}^{\prime}\right|<\varepsilon$ and $\varrho\left(x_{i}^{\prime}\right)=\varrho\left(x_{i}\right)$ for each $i=1, \ldots, k$. In other words, a Nash equilibrium is generic if, whenever we slightly vary the distribution, it is possible to find another Nash equilibrium with the same number of candidates for each of the ordered positions and positions which are very close to the initial ones. In particular, an equilibrium with $k$ policy positions is not generic if for each $\delta>0$ it is possible to find $f^{\prime} \in B_{\delta}(f)$ such that there is no equilibrium with $k$ policy positions at $f^{\prime}$.

The following fact, generalizing an observation already made in Osborne (1993) and Osborne and Slivinski (1996), will be useful in identifying nongeneric equilibria.

Fact 1. Fix a divergent policy profile $\mathcal{X}=\left(\left\{x_{1}, \ldots, x_{k}\right\}, \varrho\right)$. Evenness and at least one of the following points:

1. there exists $1<i<k$ such that $\sigma_{1}\left(x_{i}\right)=\sigma_{\mathrm{r}}\left(x_{i}\right)$ and $x_{i}-x_{i-1}=$ $x_{i+1}-x_{i}$,

2. there exists $1 \leq i<j \leq k$ such that $\sigma_{\mathrm{l}}\left(x_{i}\right)=\sigma_{\mathrm{r}}\left(x_{i}\right)$ and $\sigma_{\mathrm{l}}\left(x_{j}\right)=$ $\sigma_{\mathrm{r}}\left(x_{j}\right)$,

are non-generic properties of the distribution.

In other words, given a divergent policy profile, any distribution $F$ under which this policy profile is even and either has an internal policy with a balanced support of equal lengths of left and right constituencies, or has two different policies, each of them with a balanced support, is non-generic.

\section{Equilibrium characterisation}

There are some obvious properties that must be satisfied by any equilibrium configuration. Clearly, it can't be the case that there are no candidates, since 
in that case any player can profitably deviate by entering and winning the election with probability 1 . Furthermore, given the way in which we defined the preferences, each entrant must have a strictly positive probability of winning. The following lemma states some straightforward properties of any Nash equilibrium.

Lemma 1. Let $s$ be a Nash equilibrium with policy profile $\mathcal{X}_{s}=\left(\left\{x_{1}, \ldots, x_{k}\right\}, \varrho\right)$. Then:

1. $k \geq 1$ and the probability of victory for each $i \in N$ such that $s_{i} \neq$ Out is strictly positive.

2. If exactly two candidates enter, then they must tie in the first round. If three or more candidates enter, then either they all tie in the first round or one candidate gets the highest vote share in the first round while all other candidates tie for the second place.

3. If $k \geq 2$, then $x_{1}<m<x_{k}$.

Point 1 follows directly from our assumption on the players' preferences. Point 2 also follows from the fact that all the candidates entering the contest must have a strictly positive probability of winning. Thus, each candidate must be at least second in the first round in order to have a positive chance to go to the second round. Point 3 proves the fairly intuitive result that whenever at least two positions are occupied they cannot be on the same side of the median. If all candidates are on one side of the median, say $x_{1} \geq m$, then there must be multiple candidates at the extreme position $x_{k}$. If not, the lone candidate at $x_{k}$ would lose with probability one in any runoff and it would be better off choosing Out. But if there are multiple candidates at $x_{k}$ it is easy to show that any of them can profitably deviate to $m-\varepsilon$, for some $\varepsilon$ sufficiently small, and win the election with probability 1.

The rest of the section will establish further properties that a Nash equilibrium must satisfy. In Proposition 1 we will develop some general properties of the equilibria, while Proposition 2 is devoted to the analysis of equilibria in which multiple policies are proposed. In the next section we will show that equilibria, both convergent and divergent, do in fact exist.

Proposition 1. Suppose $\mathcal{X}_{s}=\left(\left\{x_{1}, \ldots, x_{k}\right\}, \varrho\right)$ is a policy profile induced by a Nash equilibrium s. Then:

1. Whenever $k \geq 2$, extreme policies must be proposed by at least two candidates.

2. $\mathcal{X}_{s}$ is even, so that all candidates tie for first place in the first round.

3. Whenever $k \geq 3$, the probability of winning the election by a candidate is strictly decreasing in the distance of his policy from the median. 
Extreme policies must be proposed by multiple candidates since otherwise a single candidate would benefit from moving towards the median. ${ }^{9}$ This fact drives all the convergence results in HD models and it is also present for FPTP. That in equilibrium all candidates must tie in the first round, is obvious when exactly two candidates enter. When more than two candidates enter, it has to be the case that every candidate has a chance of winning, and hence of moving to the second round. We know by Lemma 1 that either all candidates tie or there is a single candidate with strictly highest vote share, say candidate $w$ at position $x_{w}$, and all the others tie for second place. The winner must be the only one occupying her policy position (since she is not tying) and thus it cannot occupy an extreme position, since we have established that such positions are occupied by at least two candidates. Furthermore, $w$ goes to the second round with probability 1 , since it is the unique winner at the first round. But this implies that the extremist candidates on the same side of the median as $w$ have no chance of winning the election. Thus the proposed policy profile cannot be the result of a Nash equilibrium. Finally, since all candidates have the same vote share in the first round, each pair of candidates has an equal probability of going to the second round, where the candidate closer to the median is bound to win or each candidate wins with probability $\frac{1}{2}$ if their distance from the median is the same. Thus, a candidate closer to the median is more likely to be matched with an opponent who is more far away from it.

The property that extreme policies must be occupied by multiple candidates holds also under the FPTP rule. However, under FPTP there is an incentive for candidates to move away from the median if there are already multiple candidates at that position and the constituency on at least one side of the median is larger than the share of votes obtained by each candidate proposing the policy. For this reason extreme policies have to be proposed by at most two candidates and have to have balanced constituencies. The implication of this, as shown by Osborne (1993), is that divergent equilibria, although possible, are non-generic under the FPTP rule.

Under the runoff rule extreme policies also have to be proposed by multiple candidates, but moving further away from the median is costly, as it leads to losing the second round. This means that extreme policies cannot exceed certain bounds. The next proposition states the properties of equilibria in which multiple policy positions are taken.

For any policy profile $\mathcal{X}_{s}=(X, \varrho)$, with $X=\left\{x_{1}, \ldots, x_{k}\right\}$, if $k \geq 3$, call internal a policy $x_{i}$ such that $i \notin\{1, k\}$.

\footnotetext{
${ }^{9}$ Callander (2005) finds an equilibrium in which only one candidate enters at each location. The existence of these equilibria is due to the different game form that he considers, with a potential entrant moving after the two incumbents. The reason why a candidate does not want to move towards the median is that this ends up inducing entry. We instead consider a game with simultaneous entry, and in this game there are no equilibria in which only two players enter.
} 
Proposition 2. Let $s$ be a Nash equilibrium and let $\mathcal{X}_{s}=(X, \varrho)$, with $X=\left\{x_{1}, \ldots, x_{k}\right\}$ and $k \geq 2$. Then:

1. $q_{\underline{\underline{\rho\left(x_{1}\right)-1}}}^{\underline{\varrho\left(\mathcal{X}_{s}\right)}} \leq x_{1}$ and $x_{k} \leq q_{\underline{\underline{\rho\left(\mathcal{X}_{s}\right)-\varrho\left(x_{k}\right)+1}}}$. Moreover, either the extreme policies are symmetric about the median or one of them is proposed by exactly two candidates, has balanced constituency and is strictly closer to the median than the other one.

2. If $k \geq 3$, then the median voter never votes for an extreme policy.

3. If $k \geq 3$, then all the internal policies are proposed by at most two candidates, and if such a policy is proposed by two candidates, then its support is balanced. Moreover, no internal policy proposed by exactly one candidate may lie between two binary policies with balanced support.

In the case of internal (i.e. not extreme) policies, their basic properties under runoff are like under FPTP system. Each such policy is at most binary and those of them that are binary must have a balanced support. The fact that the mass of both left and right constituency of such policies is restricted to be no larger than the share of votes obtained by each of the proposing candidates has the same reasons in the case of both systems: there is an incentive for candidates proposing such policies to move towards the constituency larger than this share. Additionally a unary policy can never lie between two binary policies with balanced constituency. This property follows from the assumption that players are purely office-seeking. If a candidate proposes his policy alone and his policy is between two binary policies with balanced constituencies, then there is always a profitable deviation for him within the interval bounded by those two policies. This is true in the case of FPTP system as well and it implies, in particular, that all three-policy equilibria must have each of the policies proposed by exactly two candidates under that system. ${ }^{10}$

\section{Existence of equilibria}

In this section we discuss the existence of both convergent and divergent equilibria. As pointed out in the introduction, under FPTP the only case in which an equilibrium generically exists is when $n=2$, and in that case the only equilibrium is convergent. Haan and Volkerink (2001) have shown that under the runoff rule there are convergent equilibria for any $n$, and that convergent equilibria are such that all players enter and position themselves at the median.

\footnotetext{
${ }^{10}$ This fact is not shown in Osborne (1993), but was already observed in Dziubiński and Roy (2009).
} 
Proposition 3 (Haan and Volkerink). Let $n \geq 2$ be the number of players. Then there always exists an equilibrium in which all players enter at the median. This is the only convergent equilibrium.

That having all candidates positioned at the median is an equilibrium follows from the fact that whenever a candidate moves away from the median then she can improve (for $n \geq 3$ ) her vote share in the first period, but then in the second period she will lose for sure because she will be matched with a candidate located at the median. Notice that having $n^{*}<n$ candidates entering cannot be an equilibrium, since any of the players choosing Out could enter at the median and win with probability $\frac{1}{n^{*}+1}$. Also, no point different from the median can be occupied in a convergent equilibrium, since in that case moving to the median would guarantee outright victory in the first round.

Proposition 1 implies that the existence of a divergent equilibrium requires at least four players, as there are at least two extreme positions and each of them must be occupied by at least two candidates. Combining this with Proposition 3 we have the following corollary.

Corollary 1. If $n=3$ then the only equilibrium is convergent. All players enter and position themselves at the median.

Proposition 3 shows that the HD model is not plagued by non-existence when runoff, rather than FPTP, is used. What about uniqueness? For $n=2$ runoff is equivalent to FPTP, so the median voter theorem applies. Corollary 1 shows that the median voter theorem is preserved for $n=3$.

When $n \geq 4$ divergent equilibria appear and the rest of this section will be devoted to analysing such equilibria. Such possibility was already observed by Callander (2005) for the case of bell-shaped distributions symmetric about the median. To be sure, in the case of symmetric distributions, two policy equilibria exist also under FPTP. However such equilibria are not generic, as shown in Osborne (1993). We show that they are possible for any non-atomic distribution and fully characterize the set of two-policy equilibria. As we show below, in all two-policy equilibria the platforms proposed must be symmetric about the median and each of them must be proposed by the same number of candidates. By Proposition 1 this number must be at least two. These features are similar to those observed by Callander (2005) for the case of symmetric distributions.

Let $r \geq 2$ and consider a policy profile where $m-e$ and $m+e$ are the only policies proposed and each of them is proposed by $r$ candidates. By point 1 of Proposition 2 we know already that extreme policies cannot be too far from the median, if this is to be an equilibrium policy profile. This is related to the threat of inward deviations by the candidates proposing extreme policies. Thus there is a threshold value $d^{r}(F)$ such that a candidate proposing one of the extreme positions could profitably deviate inwards iff 
$e \geq d^{r}(F)$. For $r \geq 3, d^{r}(F)$ is the minimum value such that if $e=d^{r}(F)$, then a candidate proposing an extreme position could deviate inwards and tie for the first place. For $r=2$ this is insufficient, because a deviation inwards could be profitable even if the deviating candidate lost but tied for the second place in the first round. In this case $d^{r}(F)$ is defined to be the minimum value such that if $e=d^{r}(F)$, then a candidate proposing an extreme position could deviate inwards and tie for the second place. It is easy to see that for any distribution $F$ and for any $r \geq 2$, it must be that $d^{r}(F)>0$.

Additionally, if such equilibria are also robust to free entry (that is, if there are players who stay out of the elections, a case possible only if $n>4$ ), then the requirement to block entry also affects the distance between the two policies. For any given policy configuration $\{m-e, m+e\}$, the vote share of a player located at $y \in(m-e, m+e)$ is the same, whether this player is a new entrant or a repositioning old candidate. However, in the case of the new entrant the remaining votes are split among $n$ candidates while in the case of a deviator they are split among $n-1$ candidates. Thus, new entry is typically more dangerous for an equilibrium than a deviation and the conditions to make sure that a configuration is an equilibrium are stricter when some players stay out of the competition. In other words, equilibria in which some players stay out typically have positions closer to the median than equilibria in which all players enter.

As we pointed out already, under runoff the threat of entry applies only to the area between the two policies. Let $e^{r}(F)>0$ be the minimal value such that if $e \geq e^{r}(F)$, then there is a position within $(m-e, m+e)$ such that a player could enter there and gain the support $\frac{1}{2 r+1}$.

We are now ready to state the following proposition which shows that two-policy equilibria always exist provided $n \geq 4$. Moreover, it gives a complete characterisation in the case where the number of candidates is less then the number of players or when $n=4$.

Proposition 4. Let $n \geq 4$. Then for any non-atomic distribution $F$ with unique median $m$, any $2 \leq r \leq n / 2$ and any $0<e<\min \left\{e^{r}(F), d^{r}(F)\right\}$ there exists a two-policy Nash equilibrium with policy profile $\mathcal{X}_{s}=(\{m-$ $e, m+e\}, \varrho)$ and $\varrho(m-e)=\varrho(m+e)=r$. Moreover, if $s$ is a Nash equilibrium with policy profile $\mathcal{X}_{s}=\left(\left\{x_{1}, x_{2}\right\}, \varrho\right)$, then the following hold:

1. $x_{1}=m-e$ and $x_{2}=m+e$, for some $e>0$.

2. $n \geq 4$ and $\varrho\left(x_{1}\right)=\varrho\left(x_{2}\right) \geq 2$.

3. $e<d^{r}(F)$.

4. If some players stay out of competition, that is $\varrho\left(\mathcal{X}_{s}\right)<n$, then $e<$ $e^{r}(F)$. 
Notice that for $r=2$ it holds that $d^{r}(F) \leq e^{r}(F)$. This is because if there is a position $t \in(m-e, m+e)$ that allows a player staying out of the elections to enter and tie for the first place, then a candidate from one of the extreme positions could deviate to $t$, which would always guarantee him going to the second round with a probability of at least $1 / 3$ (where he would win). Hence his overall probability of winning the elections increases above $1 / 4$, the probability with which he was winning originally. Thus for $r=2$ point 4 is automatically guaranteed. For $r \geq 3$, on the other hand, it holds that $d^{r}(F) \geq e^{r}(F)$. This is because if there is a profitable deviation to some position $t \in(m-e, m+e)$ by a candidate proposing one of the extreme policies, then a deviator must at least tie for the first place. In particular, he must tie with the candidates proposing his original position. Thus if a candidate staying out of the elections would enter at $t$, then he would tie with candidates proposing one of the extreme positions and defeat all the candidates proposing the other extreme position. Hence for $r \geq 3$ both points 3 and 4 are needed.

Remark 1. Convergent equilibria are always such that all players enter at the median. One implication is that this type of equilibria cannot survive free entry, as the number of candidates would go to infinity (here we assume that only equilibria with a finite number of candidates are interesting). By contrast, divergent equilibria do not suffer from this shortcoming. As long as $n>4$ there are always divergent equilibria in which at least one player decides to stay out. Adding additional players does not change the basic incentives of the equilibrium: each additional potential entrant will still find optimal to stay out (this is similar to Callander (2005), but he assumes that an entrant located at the same position as an incumbent gets a vote share of zero). It should be pointed out, however, that the non-existence problem for the convergent equilibrium disappears if there is a strictly positive cost of entry. Denoting $b$ the benefit of winning the election and $c$ the cost of participation, when there is free entry it is an equilibrium that exactly $n^{*}<n$ candidates enter at the median, where $n^{*}$ is such that $\frac{b}{n^{*}+1}<c \leq \frac{b}{n^{*}}$.

\section{Equilibria with three or more policies}

Equilibria with more than two policies are generically possible, however unlike the two-policy case, not every non-atomic distribution can support them. In this section we give characterisation of such equilibria and discuss the properties of distributions that allow for their existence.

\subsection{Three-policy equilibria}

As we explained in Section 4, the only three-policy equilibria that are possible under FPTP rule with purely office-seeking players must have each of the 
policies proposed by exactly two candidates. Moreover, such equilibria are non-generic. In this section we show that in the case of runoff system three policy equilibria are generically possible. Moreover, in any generic case the middle policy is always proposed by a single candidate, while extreme positions are symmetric about the median and are proposed by an equal number of candidates. Depending on the properties of the distribution many such equilibria can exist. However, there can be at most one such equilibrium for any given number of candidates.

In Callander (2005) it is shown that three-policy equilibria are possible under runoff if the distribution is symmetric about the median and bell-shaped. Equilibrium considered there have extreme policies symmetric about the median and exactly one candidate proposing $m$. Interestingly, this symmetry is not due to symmetry of the distribution. As we show, all the generic three-policy equilibria have extreme policies symmetric about the median. However, the candidate proposing the internal policy is never proposing $m$ in any generic case. In the case of distributions considered in Callander (2005) it is also possible to have three-policy equilibria with each of the three-policies proposed by exactly two candidates. As we show below such equilibria are not generic (note that symmetry of the distribution is not a generic property).

We start with identifying the properties that have to be satisfied by any generic three-policy equilibrium. These properties are pretty sharp: generic three policy equilibria must have exactly one candidate at the internal policy which is never the median voters' ideal point, the same number of candidates at the extreme positions and have extreme policies symmetric around the median.

Proposition 5. Let $n$ be the number of players and suppose that $s$ is a generic Nash equilibrium with policy profile $\mathcal{X}_{s}=\left(\left\{x_{1}, x_{2}, x_{3}\right\}, \varrho\right)$. Then the following hold

1. $\varrho\left(x_{1}\right)=\varrho\left(x_{3}\right)=r$ with $2 r+1 \leq n$.

2. $x_{1}=m+q_{\frac{r}{2 r+1}}-q_{\frac{r+1}{2 r+1}}, x_{3}=m-q_{\frac{r}{2 r+1}}+q_{\frac{r+1}{2 r+1}}$ and $x_{2}=q_{\frac{r}{2 r+1}}+$ $q_{\frac{r+1}{2 r+1}}-m$.

3. $\varrho\left(x_{2}\right)=1$ and $\varrho\left(x_{1}\right)=\varrho\left(x_{3}\right) \geq 2$.

Proposition 5 establishes the properties of generic three-policy equilibria. The question is whether such equilibria can exist at all. In Proposition 6 below we give necessary and sufficient conditions that a distribution has to satisfy to allow for existence of a three-policy equilibrium with $c$ candidates. The conditions we give are for the case of equilibria with some of the players staying out of the elections (that is $c<n$ ). In the case of full participation equilibria (when $c=n$ ) these conditions can be weakened, as remarked after the proposition. 
Proposition 5 specifies exactly a three-policy profile with $c=2 r+1$ candidates. To guarantee that this profile is an equilibrium policy profile, the distribution has to be such that the following deviations from the players are not profitable:

(a). repositioning by the candidate proposing $x_{2}$ within the interval $\left(x_{1}, x_{3}\right)$,

(b). new entry in the interval $\left(x_{1}, x_{2}\right)$ or in the interval $\left(x_{2}, x_{3}\right)$.

(c). repositioning by a candidate proposing $x_{1}$ in the interval $\left(x_{1}, x_{2}\right)$ and repositioning by a candidate proposing $x_{3}$ in the interval $\left(x_{2}, x_{3}\right)$,

All the remaining possible deviations are either never profitable or are not profitable if the deviations above are ruled out. Moreover, if there are players staying out of the elections, then ruling out entry is enough to rule out repositioning by the candidates proposing extreme positions. We now move to define a class of distribution functions that will be necessary and sufficient to block these deviations.

For any integer $r \geq 2$, let $\mathcal{F}_{r}$ be a class of densities such that for each $f \in \mathcal{F}_{r}$ with median $m$ and its corresponding distribution $F$, the following properties hold:

1. for all $t \in\left(0, m-q \frac{r}{2 r+1}\right)$,

$$
F\left(q_{\frac{r+1}{2 r+1}}+t\right)<\left(\frac{r+1}{r}\right) F\left(q_{\frac{r}{2 r+1}}+t\right)
$$

2. for all $t \in\left(0, q_{\frac{r+1}{2 r+1}}-m\right)$,

$$
1-F\left(q_{\frac{r}{2 r+1}}-t\right)<\left(\frac{r+1}{r}\right)\left(1-F\left(q_{\frac{r+1}{2 r+1}}-t\right)\right),
$$

3. for all $t \in\left(m+q_{\frac{r}{2 r+1}}-q_{\frac{r+1}{2 r+1}}, q_{\frac{r}{2 r+1}}\right)$,

$$
F\left(t+q_{\frac{r+1}{2 r+1}}-m\right)-F(t)<\frac{1}{2 r+1},
$$

4. for all $t \in\left(q_{\frac{r}{2 r+1}}+q_{\frac{r+1}{2 r+1}}-m, q_{\frac{r+1}{2 r+1}}\right)$,

$$
F\left(t+m-q_{\frac{r}{2 r+1}}\right)-F(t)<\frac{1}{2 r+1},
$$


Notice that the restrictions imposed by conditions $1-4$ leave some scope of freedom and so they are robust to small perturbations of the distributions satisfying them (hence they are generic). For example, they are satisfied by any density which is sufficiently close to uniform within the interval $\left(x_{1}, x_{3}\right)$. Similarly, densities sufficiently close to the $f$ with $f(t)>f(u)$, for all $t \in$ $\left(q_{\frac{r}{2 r+1}}, q_{\frac{r+1}{2 r+1}}\right)$ and $u \in\left(x_{1}, q_{\frac{r}{2 r+1}}\right) \cup\left(q_{\frac{r+1}{2 r+1}}, x_{3}\right)$ and with the mass sufficiently evenly distributed with the interval $\left(q_{\frac{r}{2 r+1}}, q_{\frac{r+1}{2 r+1}}\right)$ (so that points $3-4$ hold) satisfy this property. There are even densities with $f(t)<f(u)$ (where $t$ and $u$ are as above) that satisfy these properties, although in this case the densities must be sufficiently close to uniform within the interval $\left(x_{1}, x_{3}\right)$.

We have the following proposition.

Proposition 6. Let $r \geq 2$ and $n>2 r+1$. Then there exists a Nash equilibrium with policy profile $\mathcal{X}_{s}=\left(\left\{x_{1}, x_{2}, x_{3}\right\}\right.$, @) such that $m-x_{1}=$ $x_{3}-m, \varrho\left(x_{2}\right)=1$ and $\varrho\left(x_{1}\right)=\varrho\left(x_{3}\right)=r$ if and only if the voters' ideologies are distributed according to some density $f \in \mathcal{F}_{r}$. Consequently, this Nash equilibrium is generic.

For $r>2$ and $n=2 r+1$ conditions $1-2$ are necessary, while conditions 3 and 4 are sufficient but not necessary for the result to hold. In this case there is no threat of entry from a player who stays out of the elections and weaker conditions are needed to prevent deviations by candidates proposing extreme positions to positions within intervals $\left(x_{1}, x_{2}\right)$ and $\left(x_{2}, x_{3}\right)$.

It is also important to note that the properties $1-4$ are of a local nature in the sense that they concern the distribution within the interval $\left(x_{1}, x_{3}\right)$. Both pairs of conditions, $(1-2$ and $3-4)$ limit the speed of change of $F$ at different intervals within $\left(x_{1}, x_{3}\right)$. This is needed to block profitable deviations within the interval $\left(x_{1}, x_{3}\right)$ of the candidate proposing $x_{2}$ (conditions 1 -2 ), as well as the threat of entry in this interval (conditions $3-4$ ). Notice that conditions $3-4$ imply, in particular, that $q_{\frac{r}{2 r+1}}-q_{\frac{r-1}{2 r+1}} \geq q_{\frac{r+1}{2 r+1}}-m$ and $q_{\frac{r+2}{2 r+1}}-q_{\frac{r+1}{2 r+1}} \geq m-q_{\frac{r}{2 r+1}}$, so that $q_{\frac{r-1}{2 r+1}} \leq x_{1}$ and $x_{3} \leq q_{\frac{r+2}{2 r+1}}$.

\subsection{Equilibria with more than three policies}

Equilibria with more than three policies can exist only if $n \geq 6$. It turns out that in any generic configuration of this kind internal policies are proposed by exactly one candidate. Hence, unariness of internal policies is indeed a general feature of any divergent equilibrium. Moreover, if the number of policies is even, then one of the extreme policies must be proposed by exactly two candidates. In this case the two extreme policies are not symmetric about the median and the one closer to the median has a balanced constituency. If the number of policies is odd then both cases, of extreme policies being symmetric about the median and of extreme policies being 
non-symmetric about the median, are possible. We also show that the policies proposed in any generic equilibrium are uniquely determined by the distribution, the number of policies and the number of candidates (we omit the formulas specifing this uniqueness relation in the proposition below for the sake of clarity, and move them to the Appendix, where the following proposition is proven).

Proposition 7. Let $n$ be the number of players and suppose that $s$ is a Nash equilibrium with policy profile $\mathcal{X}_{s}=\left(\left\{x_{1}, \ldots, x_{k}\right\}, \varrho\right)$, where $\varrho\left(\mathcal{X}_{s}\right)=c$ and $k \geq 4$. Then the following holds in any generic case:

1. The values of $x_{1}, \ldots, x_{k}$ are uniquely determined by $F, k$ and $c$.

2. For all $i \in\{2, \ldots, k-1\}, \varrho\left(x_{i}\right)=1$.

3. If $k$ is even, then $m-x_{1} \neq x_{k}-m$. Moreover, if $m-x_{1}<x_{k}-m$, then $\varrho\left(x_{1}\right)=2$ and $x_{1}$ has a balanced support, and if $m-x_{1}>x_{k}-m$, then $\varrho\left(x_{k}\right)=2$ and $x_{k}$ has a balanced support.

As in the case of three-policy equilibria, the $k$-policy profiles with $k \geq 4$ and $c$ candidates are determined uniquely by the distribution. To guarantee that these profiles are equilibrium policy profiles, the distribution has to satisfy several properties guaranteeing that no deviations by the players are possible in the associated strategy profiles. It is sufficient and necessary to rule out the following deviations:

(a). for each $3 \leq i \leq k-2$, repositioning by the candidate proposing $x_{i}$ within the interval $\left(x_{i-1}, x_{i+1}\right)$,

(b). new entry in the interval $\left(x_{1}, x_{3}\right)$ or in the interval $\left(x_{k-2}, x_{k}\right)$.

(c). repositioning by a candidate proposing $x_{1}$ in the interval $\left(x_{1}, x_{2}\right)$ and repositioning by a candidate proposing $x_{k}$ in the interval $\left(x_{k-1}, x_{k}\right)$,

Notice that if a deviation by a candidate proposing an internal policy $x_{i}<m$, with $3 \leq i \leq k-2$, to some position $t \in\left(x_{i}, \min \left(x_{i+1}, m\right)\right)$ is not profitable, then there is no profitable entry by a player staying out of the elections possible within this interval. This is because if such an entry was possible, then the entrant would have to obtain at least the same support as that obtained by each of the standing candidates. But then the candidate proposing $x_{i}$ could also deviate to the point of entry, making his left neighbour win and his right neighbour lose the first round. Such a deviation would guarantee winning the second round, upon being selected in the first round. The probability of winning the elections by the deviator would increase from $\frac{1}{c}$ to $\frac{1}{c-2}$. Similar observation holds for $x_{i}>m$, with $3 \leq i \leq k-2$, and deviation to some position $t \in\left(\max \left(x_{i-1}, m\right), x_{i}\right)$.

As we discussed in the case with three-policy equilibria, entry at positions already taken by candidates or outside the external policies is never 
profitable. It is also not profitable for a candidate not to enter, if he has a chance of winning the elections. Also a deviation by a candidate proposing an internal policy to a position which is outside the interval bounded by his neighbouring policies is never profitable if entry at such positions is not profitable. Thus ruling out the deviations (a) - (c) is indeed sufficient and necessary to guarantee that the policy profiles under consideration are equilibrium policy profiles.

Formal constraints on the distributions that are sufficient and necessary to rule out the deviations (a) - (c) are similar to those given in the case of three-policy equilibria and we will focus on the constraints ruling out deviations given in point (a) only. Suppose that $3 \leq i \leq k-2$ and that $x_{i}<m$ (the case $x_{i}>m$ is symmetric). A deviation to some $t \in\left(x_{i-1}, x_{i}\right)$ is profitable if the deviator obtains a mass of votes which is larger than the mass of votes gained by his right neighbour after the deviation. Thus to rule out such a deviation, the distribution must satisfy, for all $t \in\left(0, x_{i}-b_{i-1}\right)$,

$$
F\left(b_{i-1}\right)-F\left(b_{i-1}-t\right)<2\left(F\left(b_{i}\right)-F\left(b_{i}+t\right)\right) .
$$

A deviation to some $t \in\left(x_{i}, x_{i+1}\right)$ is profitable if it does not decrease the mass of votes of the deviator. This is because, as we explained above, such a deviation would make the left neighbour win the first round, the right neighbour lose the first round and increase the probability of winning the elections by the deviator. Thus to rule out such a deviation, the distribution must satisfy, for all $t \in\left(0, b_{i}-x_{i}\right)$,

$$
F\left(b_{i-1}+t\right)-F\left(b_{i-1}\right)<2\left(F\left(b_{i}+t\right)-F\left(b_{i}\right)\right) .
$$

Notice that the density function $f$ can be neither constant nor increasing over the interval $\left[x_{3}, m\right]$. However, a density function decreasing over this interval would satisfy the second property, and if its slope was not too large, then it would satisfy the first property. Also, distributions sufficiently close to such a distribution would satisfy the requirements.

Generally, as in the case of three-policy equilibria, the constraints on the distributions are of local nature and affect the distribution within the interval $\left(x_{1}, x_{k}\right)$ only. Moreover, they leave some scope of freedom, and can always be sustained under sufficiently small perturbations. Hence equilibria supporting $k$-policy profiles with $c$ candidates are generic, although the distributions supporting them must satisfy special properties.

We then conclude our analysis with the following observations that suggest that in the HD model a la Osborne (1993), multi-policy equilibria are less likely in an arbitrary election. Let $\left\{x_{1}, . ., x_{k}\right\}$ be the policies. Then, existence requires restrictions on the distribution only in the support $\left[x_{1}, x_{k}\right]$. These requirements are different for $k=3$ and $k>3$. However, for $k=3$, the requirements are relatively less restrictive. Distributions that do not decrease too rapidly on $\left(x_{1}, m\right)$, do not increase too rapidly on $\left(m, x_{2}\right)$, and 
do not have large concentrations of mass on small subintervals of the interval $\left(x_{1}, x_{3}\right)$ support existence. Examples of distributions that satisfy these properties are distributions that are roughly uniform or which are roughly single peaked over the interval $\left(x_{1}, x_{3}\right)$ with the peak somewhere near the median $m$. For $k>4$ the requirements are quite restrictive. Roughly speaking they require that the density function decreases (but not too fast) on the interval $\left(x_{3}, m\right)$ and increases (but again, not too fast) on the interval $\left(m, x_{k-2}\right)$. Examples of distributions that could satisfy these restrictions are bimodal distributions, with $x_{1}$ and $x_{k}$ lying on the internal slopes of different 'bumps'. The case of $k=4$ is quite delicate as well. If $m$ is closer to $x_{1}$ or $x_{2}$, then the requirements for it will be like in the case with five and more policy equilibria. The only case where $m$ is not closer to one of these two policies is where there are 2 candidates on each of the extremes. In that case $m$ is exactly on the border between $x_{1}$ and $x_{2}$.

\section{Variations}

Up to now we have analysed the benchmark case $z=50$ (i.e. a majority of votes is needed to win the first round) and the two top most voted candidates going to the second round. As pointed out in the introduction, and as discussed more at length in Grofman (2008), there are many varieties of the runoff system and one typically expects variations in the rules of the game to change the equilibrium set. In this section we discuss such changes for a couple of important variants of the benchmark case.

\subsection{Less than majority in the first round}

Some countries use a runoff rule with $z<50$. Thus, a candidate wins at the first round if its vote share is at least as high as that of any other candidate and it is at least $z \%$ of the votes. Otherwise, the two top vote-getters go to the second round where the election is decided by FPTP.

One first consequence of this change is that the convergent equilibrium disappears. In a convergent equilibrium all $n$ players enter at the median and get a vote share of $1 / n$. When $z=50$ no profitable deviation is possible because a deviator can get a vote share arbitrary close, but inferior, to $50 \%$ and therefore the second round is still triggered where the deviator loses with certainty to one of the median candidates. If $z<50$ this is no longer true. It is possible to find deviations insuring strictly more than $z \%$, so that the deviator gets elected right at the first round. This implies that in the case $z<50$ equilibria, if they exist, can only be divergent.

Most of the results on characterisation established for the classical case still hold for the case $z<50$. In fact, it can be checked that Propositions 1 and 2 hold when $z<50$. Thus, for example, divergent equilibria must have 
at least two candidates located at the extremes, since otherwise a single candidate would have an incentive to move towards the median. One immediate implication is that a divergent equilibrium requires at least 4 players. This, together with the non-existence of convergent equilibria, implies that there is no equilibrium when $n=3$.

It also remains true that equilibria with more than two policies can exist generically, essentially for the same arguments proposed in the benchmark case. For two-policy equilibria, unlike for the case with $z=50$, existence is however no longer guaranteed when $z<50$. To see this, consider a configuration in which $r$ players enter at $m-e$ and another $r$ players enter at $m+e$. In the classic case we never have to worry about entry (by a new candidate or a deviator) on the wings, i.e. at locations $y<m-e$ or $y>m+e$. The reason is that no deviator in that region can get more than $50 \%$ of the vote in the first round and it would face sure defeat in the second round. When $z<50$ deviations on the wings may become profitable and thus may destroy a two-policy equilibria. The logic is the same by which $z<50$ implies that a convergent equilibrium is possible. For example, it is clear that no two-policy equilibrium in which not all players enter is possible if $F(m-e)>z$ or $1-F(m+e)>z$, since an entrant could enter on the wing and win the election in the first round ${ }^{11}$. It is easy to see how this can lead to non-existence. In fact, in order to prevent profitable entry in the interval $(m-e, m+e)$ the value of $e$ cannot be too large, but in order to prevent profitable entry outside that interval the value of $e$ cannot be too small. The two requirements become incompatible when $z$ is sufficiently low. When $z$ approaches zero then runoff becomes essentially indistinguishable from FPTP, thus it has all the existence problems of FPTP. Even when equilibria exists (for $z$ sufficiently close to 50 ) there will be a lower bound on $e$. Thus, smaller is $z$ greater is the scope of extremism.

The case $z>50$ is not interesting as it is equivalent to the case $z=50$. This follows from the fact that in equilibrium no candidate can get strictly more than $50 \%$ of the vote at the first round, no matter what the value of $z$ is. If a candidate gets more than $50 \%$ at the first round then either it wins outright or it moves with probability 1 to the second round where it wins with probability 1 , as its vote share cannot decrease when other candidates drop out. This implies that no other candidate should enter, since it would lose with probability one, so the equilibrium has a single entrant. This is impossible, since another candidate can enter exactly at the same position, get $50 \%$ of the vote and win the election with probability $\frac{1}{2}$. Thus, for each $z \geq 50$ all equilibria have at least two entrants and each entrant gets no more than $50 \%$ of the vote at the first round. This implies that the first

\footnotetext{
${ }^{11}$ To be precise, it has also to be the case that the entrant gets a vote share greater than any other existing candidate. This is surely the case whenever $z>25$; since at least 4 players enter and must tie, each of the existing candidates must have less than $25 \%$ of the vote.
} 
round threshold is irrelevant, as long as it is not lower than 50 .

\subsection{More than two candidates in the second round}

Existence problems may also appear when more than two candidates are admitted to the second round. To fix ideas, consider the rule used in the French legislative elections that we first describe. Any candidate that gets a majority in round 1 wins outright; if that is not the case, then any candidate that gets at least $12.5 \%$ of the votes in round 1 goes to round 2 ; if there are exactly two candidates that get more than $12.5 \%$ in round 1 , then only these two candidates move to round 2 ; if exactly one candidate gets more than $12.5 \%$, then it goes to round 2 while exactly one candidate out of the 2nd highest shares is randomly chosen to go to round 2 and these are the only two candidates in round 2 ; finally, if all candidates obtain less than $12.5 \%$, then only two top voted candidates go to round 2 .

In this case there is no convergent equilibrium for $n \leq 5$. To see this, suppose that five candidates are located at the median and get $20 \%$ of the vote each. In this case the second round is a repetition of the first (all candidates go to the second round) and each candidate wins with probability $1 / 5$. Consider now a deviation to $m-\varepsilon$ by one candidate, with $\varepsilon$ small. That candidate gets slightly less than $50 \%$ of the vote, while the remaining four candidates share the remaining votes an get (slightly more than) $12.5 \%$ . Thus it remains true that all candidates move to the second round. As a consequence the deviator wins the election and this implies that having all candidates at the median is not an equilibrium ${ }^{12}$. When $n>5$ a convergent equilibrium may or may not exist, depending on the distribution. For example, consider the case $n=6$ and the uniform distribution on $[0,1]$. If all candidates are located at the median and a deviator moves slightly to the left or the right then the five candidates remaining at the median get about $10 \%$ of the vote. With a threshold of 12.5 , only one of the candidates is randomly selected for the second round, where it proceeds to defeats the deviator. However, suppose that the deviator moves far away from the median, say to $x=0$. In that case the deviator gets $25 \%$ of the vote and each of the candidates remaining at the median get $15 \%$. Thus, all candidates go to the second round where the deviator wins. Thus in this case there is no convergent equilibrium with $n=6$. In general, for a threshold of 12.5 a convergent equilibrium always exists when there are at least 9 candidates, since after the deviation there will be 8 candidates sharing the constituency

\footnotetext{
${ }^{12}$ Things get more complicated when candidates are allowed to withdraw from the second round. In the example just discussed the 4 candidates located at the median know that they are going to lose for sure if at least two remain in the second round. Thus, we can specify subgame perfect equilibria in which, say, in these situations only the candidate at the median with the lowest index does not withdraw. In this case the convergent equilibrium is restored.
} 
of the median and they cannot possibly get more than $12.5 \%$ each. Thus in that case a deviator is matched with a randomly chosen median candidate, implying defeat for the deviator.

The general issue that appears from this discussion is that in the case in which all the candidates above a certain threshold are admitted to the second round, we again have to worry, as in the case $z<50$, about deviations that move a candidate away from the median to increase the vote share in the first round. The twist in this case is that the deviator does not want to be 'too successful', since it is important to make sure that the opponents are able to get enough votes and make it to the second round, where they will split the vote and let the deviator win.

While we have illustrated these problems with reference to convergent equilibria, things are not different when we consider two-policy equilibria. We already know that the policy positions in those equilibria cannot be too far from the median, since profitable entry at the centre would become possible. When deviations away from the median may be profitable, as in the case under discussion, the policy positions can't be too close to the median either. Thus, existence problems may appear.

\section{Conclusions}

We have provided a complete characterisation of the set of Nash equilibria in the model of Osborne (1993) for the runoff system. We have shown that both convergent and divergent equilibria exist for any distribution of voters' ideal policies and for any number of players. However, the most prominent equilibria are those with two policies. This is because free entry destroys convergent equilibria, divergent equilibria are robust to free entry and equilibria with more than two policies do not always exist while those with two policies always do.

In any equilibrium, extreme policies are proposed by at least two players and all players who contest must obtain the same mass of votes in round 1 of the system. Although runoff supports many policy equilibria, there is a strong tendency that policies near the median voter's ideal point are most likely to win, hence, runoff promotes centrism. Multipolicy equilibria generically exist as well, and we observe some interesting generic properties there. Firstly, in every three policy equilibrium, the middle policy is generically different from the median while the extreme policies are equidistant from the median. Next, all internal policies are proposed by exactly one player while extreme policies need to necessarily be equidistant about the median unless there are an even number of policies upon contest.

As documented in Blais et al. (1997), the runoff is used in 49 democratic countries while the FPTP system is used in only about 20. This itself suggests the importance of runoff. Our paper fills an important theoretical 
gap in the existing literature. As we have pointed out before, there is a growing literature on this voting system. Some interesting extensions are immediate. It would be good to study the sequential model with endogenous entry timings as in Osborne (1993) to see how the runoff compares in that game with the FPTP that was studied there. It would also be important to provide a complete characterisation of equilibria in the entry deterrence game in Callander (2005) with more than 1 potential entrant. Uncertainty of the median voters' ideal policy, as in Matsushima (2007) seems another important aspect that one may wish to explore.

\section{Appendix: Proofs}

Proof of Fact 1. Let $\mathcal{X}=\left(\left\{x_{1}, \ldots, x_{k}\right\}, \varrho\right)$ be the policy profile under consideration. For point 1 take any $1<i<k$. Suppose that $\sigma_{1}\left(x_{i}\right)=\sigma_{\mathrm{r}}\left(x_{i}\right)$ and $x_{i}-x_{i-1}=x_{i+1}-x_{i}$. Since the policy profile is even, so $b_{i-1}$ is the $\sum_{j=1}^{i-1} \varrho\left(x_{j}\right)^{\prime}$ th $\varrho(\mathcal{X})$-quantile and $b_{i}$ is the $\sum_{j=1}^{i} \varrho\left(x_{j}\right)^{\prime}$ th $\varrho(\mathcal{X})$-quantile. Thus the assumption that $\sigma_{1}\left(x_{i}\right)=\sigma_{\mathrm{r}}\left(x_{i}\right)$ and $x_{i}-x_{i-1}=x_{i+1}-x_{i}$ implies

$$
\frac{q_{\frac{\varrho\left(x_{i}\right)+2 \sum_{j=1}^{i-1} \varrho\left(x_{j}\right)}{2 \varrho(X)}}}{2}-q_{\frac{\sum_{j=1}^{i-1} \varrho\left(x_{j}\right)}{\varrho(X)}}=q_{\frac{\sum_{j=1}^{i} \varrho\left(x_{j}\right)}{\varrho(X)}}-q_{\frac{\varrho\left(x_{i}\right)+2 \sum_{j=1}^{i-1} \varrho\left(x_{j}\right)}{2 \varrho(X)}}
$$

which is a non-generic property of $F$.

For point 2 suppose that policies $x_{i}$ and $x_{j}$, with $1 \leq i<j \leq k$ have balanced support. Let $d_{l}=x_{l+1}-x_{l}$, for $i \leq l \leq j-1$. Then $d_{i}=2\left(b_{i}-x_{i}\right)$ and $d_{l+1}=2\left(b_{l+1}-b_{l}\right)-d_{l}$, for $i \leq l \leq j-2$. Solving this system of equations we get

$$
d_{j-1}=2 b_{j-1}+(-1)^{j-1} x_{i}+4 \sum_{r=1}^{j-2}(-1)^{j-r-1} b_{r}
$$

On the other hand $d_{j-1}=2\left(x_{j}-b_{j-1}\right)$ and so

$$
2 x_{j}=(-1)^{j-1} x_{i}+4 \sum_{r=1}^{j-1}(-1)^{j-r-1} b_{r} .
$$

Since $x_{i}$ and $x_{j}$ have balanced support, so

$$
\begin{aligned}
& x_{i}=q^{\frac{\varrho\left(x_{i}\right)+2 \sum_{r=1}^{i-1} \varrho\left(x_{r}\right)}{2 \varrho(\mathcal{X})}} \\
& x_{j}=q_{\frac{\varrho\left(x_{j}\right)+2 \sum_{r=1}^{j-1} \varrho\left(x_{r}\right)}{2 \varrho(\mathcal{X})}} .
\end{aligned}
$$

and borders $b_{r}$ correspond to proper $\varrho(\mathcal{X})$-quantiles of $F$. Hence Equation (2) is a non-generic property of $F$.

Proof of Lemma 1. Point 1 is obvious. Point 2 is also obvious for the case of two candidates. When there are three or more candidates, each one of them must have a strictly positive probability of winning. This cannot happen if at least two, but not all, candidates tie for first place, since those who do not tie for first place would be excluded from the second round with probability 1 . Thus, either they all tie for 
first place or there is a single candidate winning the first round and all others tie for second place.

Finally, consider point 3. Suppose $k \geq 2$ and $x_{k} \leq m$ (the case $m \leq x_{1}$ is symmetric). We start noticing that in this case it must be $\sigma\left(x_{i}\right)<\frac{1}{2}$ for each $i<k$ and $\sigma_{1}\left(x_{k}\right)<\frac{1}{2}$ as well. In equilibrium a candidate located at $x_{1}$ must have a positive probability of victory. The only way in which this can happen is that the election goes to the second round and a candidate located at $x_{1}$ is matched with strictly positive probability to a candidate also located at $x_{1}$, since all other candidates are closer to the median. This implies that no candidate located at a position different from $x_{1}$ can go to the second round with probability 1 , thus all the candidates not located at $x_{1}$ either tie for first place with all other candidates or they tie for the second place with all candidates except exactly one located at $x_{1}$. The latter is impossible in equilibrium, since there must be multiple candidates located at $x_{1}$. Thus, all candidates must tie for first place. If $x_{k}<m$, then any player proposing $x_{1}$ is better off by moving to $m$, since this would give outright victory in the first round, while the probability of winning under $s$ must be strictly less than 1. If $x_{k}=m$ then it is possible to find $\varepsilon>0$ such that the constituency of $m+\varepsilon$ is strictly greater than $\max _{1 \leq i<k} \sigma\left(x_{i}\right)$ and $\sigma_{1}\left(x_{k}\right)+[F(m+\varepsilon / 2)-1 / 2]$. Hence it is profitable for a candidate proposing $x_{1}$ to move to $m+\varepsilon$. The deviation makes sure that the deviator strictly wins round 1 (with less than $50 \%$ of the vote), it increases the vote share of the candidates located at $x_{1}$ and it decreases the vote share of the candidates located at $m$. Since at the initial situation all candidates had the same vote share, in the second round the deviator faces a candidate located at $x_{1}$. Thus the deviating candidate always wins the first round and then defeats any other candidate in the second round, as she is closer to $m$

Proof of Proposition 1. Lemma 1 implies that an equilibrium policy profile is nonempty and each entrant has a strictly positive probability of victory. To see that extreme policies must be occupied by at least two candidates, suppose that $k \geq 2$ and suppose that $\varrho\left(x_{1}\right)=1$ (the case $\varrho\left(x_{k}\right)=1$ is symmetric). Let $i$ be the candidate proposing $x_{1}$. By Lemma 1 it must be $x_{1}<m$. If $x_{2}>m$, then $i$ is strictly better off by proposing $m$ instead of $x_{1}$, as this ensures victory with probability 1 . Thus, suppose $x_{2} \leq m$ and let $j$ denote a candidate proposing $x_{2}$. Since, by Lemma $1, x_{k}>m$, it must be that $k \geq 3$, i.e. at least three policy positions are occupied. Since $s$ is a Nash equilibrium, $j$ has a positive probability to go to the second round. But now observe that player $i$ is strictly better off by moving towards $x_{2}$. To see this consider two cases: (a) $i$ gets a vote share strictly larger than that obtained by the other candidates under $s$, or (b) $i$ ties with some other candidate in the first round under $s$. Suppose that case (a) holds. By Lemma 1 all the other candidates tie for the second place in the first round. Hence by moving next to the left of $x_{2}$ player $i$ rules out these candidates from the second round. This is because $k \geq 3$ and so there are other candidates to the right of $x_{2}$ who will win with those at $x_{2}$ after such a move. Moreover, the move can only increase the chances of winning with any other candidate going to the second round. Thus this move is profitable for $i$ in the case (a). Suppose that case (b) holds. In this case the above argument is even stronger as the move makes $i$ win the first round and increases his chances in the second round.

To see that all the candidates must tie in the first round, let $X_{s}=\left\{x_{1}, \ldots, x_{k}\right\}$ and $k \geq 2$ (the case $k=1$ is obvious). By Lemma 1 we know that in the first round, at least $\varrho($ mathcal $X)-1$ candidates are tied. If all of them are tied than 
we are done. Suppose then that exactly $\varrho_{s}\left(\mathcal{X}_{s}\right)-1$ of them are tied. In such a case there is a unique candidate $w$ at position $x_{w}$ with the largest mass of votes, and $\varrho\left(x_{w}\right)=1$. This candidate reaches the second stage with probability 1 . Since the extreme positions have to be occupied by at least two candidates, this candidate cannot be an extremist, i.e. $w \notin\{1, k\}$. If $x_{w} \geq m$, then all candidates at positions $x_{j}$ with $j>w$ have zero probability of victory, since they would be matched with the candidate at $x_{w}$. This cannot be an equilibrium. If $x_{w}<m$, then the argument is symmetric. We conclude that in equilibrium all candidates must tie in the first round.

Finally observe that, since in equilibrium each candidate has the same probability of getting to the second round, the probability of winning an election for candidate $i$ is equal to the probability of being matched with a candidate who is farther away from the median than $i$ plus one half the probability of being matched with a candidate who as the same distance from the median as $i$. When there are at least three positions occupied this implies that the probability of winning is strictly decreasing in the distance from the median.

Proof of Proposition 2. To prove point 1, let $s$ be a Nash equilibrium and $\mathcal{X}_{s}$ be

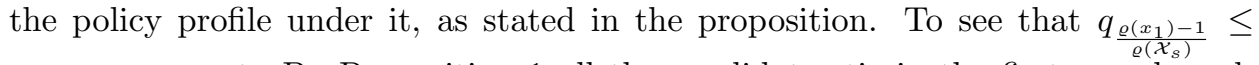
$x_{1}$, suppose not. By Proposition 1 all the candidates tie in the first round, each obtaining the support $S=1 / \varrho\left(\mathcal{X}_{s}\right)$. Since the property is violated for $x_{1}$, so $\sigma_{\mathrm{r}}\left(x_{1}\right)$ of $x_{1}$ is $>S$. But then one of the candidates proposing $x_{1}$ could deviate slightly to the right of $x_{1}$ to obtain a vote share $S^{\prime} \geq S$ (recall that by Proposition 1, $\varrho\left(x_{1}\right) \geq 2$ ). There are two cases that need to be analysed separately. Firstly suppose that $k=2$. In this case, after the deviation, all the remaining candidates proposing $x_{1}$ and the candidates proposing $x_{2}$ get their support reduced and lose the first round, while the deviator wins that round. In the second round he always wins, hence the deviation is profitable, which contradict the assumptions that $s$ is a Nash equilibrium. Secondly, suppose that $k \geq 3$. As in the previous case, all the remaining candidates proposing $x_{1}$ and the candidates proposing $x_{2}$ get their support reduced and lose the first round. Hence the only candidates that have a chance of going to the second round are those proposing the policies $x_{i}$ with $i \geq 3$ and the deviator. If $x_{2}$ is closer to the median than $x_{1}$, then the deviation is profitable for the deviator, as he rules out at least one candidate with whom he was previously losing, while he does not decrease his chances of winning in all the remaining matches. Suppose that $x_{2}-m \geq m-x_{1}$. In this case the deviator always wins in the second round, while he increases his chances of winning in the first round, ruling out the candidates proposing $x_{1}$ and $x_{2}$. Hence the deviation is profitable as well and again we get a contradiction with the assumption that $s$ is a Nash equilibrium. Arguments for the fact that $x_{k} \leq \frac{q_{\underline{\varrho\left(\mathcal{X}_{s}\right)-\varrho\left(x_{k}\right)+1}}^{\varrho\left(\mathcal{X}_{s}\right)}}{\text { are analogous }}$ and symmetric.

For the remaining part of point 1 , notice that, by what we have shown above, it holds that $\sigma_{\mathrm{r}}\left(x_{1}\right) \geq S$, with equality possible only in the case $\varrho\left(x_{1}\right)=2$, and similarly for $x_{k}$ (where $S=1 / \varrho\left(\mathcal{X}_{s}\right)$ ). Now suppose that the claim stated in that part of point 1 does not hold. Then either $m-x_{1}<x_{k}-m$ with $\sigma_{1}\left(x_{1}\right)>S$, or $m-x_{1}>x_{k}-m$ with $\sigma_{\mathrm{r}}\left(x_{k}\right)>S$. Suppose that $m-x_{1}>m-x_{k}$ with with $\sigma_{\mathrm{r}}\left(x_{k}\right)>S$ (the other case is symmetric). Then one of the candidates proposing $x_{k}$ could deviate to $x$ just to the right of $x_{k}$, so that he obtains the support $S^{\prime}>S$ and $|x-m|<\left|x_{i}-m\right|$, for all $i$ such that $x_{k}-m<\left|x_{i}-m\right|$ (notice that this is true at 
least for $i=1)$. Let $w$ denote the number of candidates proposing a policy further from $m$ than $x_{k}$ and $t$ denote the number of candidates proposing a policy different from $x_{k}$, which is in the same distance from $m$ as $x_{k}$ is. Before the deviation any candidate proposing $x_{k}$ wins with the probability

$$
\frac{w+\left(t+\varrho\left(x_{k}\right)-1\right) / 2}{\varrho\left(\mathcal{X}_{s}\right)\left(\varrho\left(\mathcal{X}_{s}\right)-1\right)}<\frac{1}{\varrho\left(\mathcal{X}_{s}\right)-1} .
$$

On the other hand, after the deviation, the deviator wins with the probability

$$
\frac{w}{\varrho\left(\mathcal{X}_{s}\right)-\varrho\left(x_{k}\right)}>\frac{1}{\varrho\left(\mathcal{X}_{s}\right)-1} .
$$

Hence there is always a profitable deviation for him, which contradicts the assumption that $s$ is a Nash equilibrium.

To prove point 2 , suppose first that $m-x_{1}=x_{k}-m$ and assume that $m \leq b_{1}$ (the case $m>b_{k-1}$ is symmetric). Then $x_{2}-m \geq m-x_{1}$ and since $k \geq 3$, so $x_{k}>x_{2}$ and, consequently, $x_{k}-m>m-x_{1}$, which contradict the assumption that $m-x_{1}=x_{k}-m$. Secondly suppose that $m-x_{1} \neq x_{k}-m$ and assume that $m-x_{1}>x_{k}-m$ (the case $m-x_{1}<x_{k}-m$ is symmetric). By point 1 , $\varrho\left(x_{k}\right)=2$. Moreover, by Proposition 1 , there are at least 5 candidates if $k \geq 3$ and each candidate obtains the same mass of votes. Thus it must be that $m<b_{k-1}$, as otherwise the candidates proposing $x_{k}$ would obtain larger mass of votes than the remaining $\geq 3$ candidates. To see that $b_{1}<m$ assume the opposite. Then $x_{2}-m \geq m-x_{1}$ and, consequently $x_{k}-m>m-x_{1}$, which contradicts our assumptions.

To prove point 3 , let $s$ be a Nash equilibrium and $\mathcal{X}_{s}$ be the policy profile under it, as stated in that point.

To see that internal policies must be at most binary and if binary, then they must have balanced constituency, assume the opposite. Then $k \geq 2$ and there exists $1<i<k$ such that either $\sigma_{1}\left(x_{i}\right)>S$ or $\sigma_{\mathrm{r}}\left(x_{i}\right)>S$, where $S=1 / \varrho\left(\mathcal{X}_{s}\right)$. Without loss of generality suppose that $x_{i} \geq m$ (the other case is symmetric). Assume also that $\sigma_{\mathrm{r}}\left(x_{i}\right)>S$ (the case of $\sigma_{\mathrm{l}}\left(x_{i}\right)>S$ can be shown by similar arguments and is easier). Since $\sigma_{\mathrm{r}}\left(x_{i}\right)>S$, so it must be that $\varrho\left(x_{i}\right) \geq 2$. Now, one of the candidates proposing $x_{i}$ just to the right and sufficiently close, so that he obtains a support $S^{\prime}>S$ and is closer to the median than any policy further from the median than $x_{i}$. Using analogous arguments to those used in proof of point 1 for extreme policy strictly closer to the median, it can be shown that this deviation is profitable, which contradicts the assumption that $s$ is a Nash equilibrium.

For the remaining part of point 3 assume the opposite, that is suppose that there is a unary policy lying between two binary policies with balanced constituency. Let this policy be $x_{i}$, with $1<i<k$. Let $S$ be the mass of support obtained by each candidate in the first round. Since both the policies $x_{i-1}$ and $x_{i+1}$ have balanced constituency and are binary, so $F\left(x_{i-1}\right)-F\left(x_{i+1}\right)=3 S$. Hence either

$$
\begin{aligned}
& F\left(\left(x_{i-1}+x_{i+1}\right) / 2\right)-F\left(x_{i-1}\right) \geq 3 S / 2 \text { or } \\
& F\left(x_{i+1}\right)-F\left(\left(x_{i-1}+x_{i+1}\right) / 2\right) \geq 3 S / 2 .
\end{aligned}
$$

Without loss of generality suppose that the first case holds. Since $F$ is non atomic and $F\left(b_{i-1}\right)-F\left(x_{i-1}\right)=S$, so there must exist $t \in\left(x_{i-1}, x_{i}\right)$ such that $F\left(b_{i-1}\right)-$ $F\left(\left(x_{i-1}+t\right) / 2\right)>3 S / 4$. We will show that if player $i$ proposed $t$ instead of $x_{i}$, 
then he would win outright. Take any $t$ satisfying the condition above and suppose that player $i$ proposed $t$ instead of $x_{i}$. Let

$$
\begin{aligned}
& S_{1}=F\left(b_{i}\right)-F\left(\left(t+x_{i+1}\right) / 2\right) \text { and } \\
& S_{2}=F\left(b_{i-1}\right)-F\left(\left(x_{i-1}+t\right) / 2\right) .
\end{aligned}
$$

By the construction above it holds that $S_{2}>3 S / 4$. Moreover, since

$$
\left(x_{i-1}+x_{i+1}\right) / 2<\left(t+x_{i+1}\right) / 2<b_{i}, \quad \text { so }
$$

$F\left(x_{i+1}\right)-F\left(\left(x_{i-1}+x_{i+1}\right) / 2\right) \leq 3 S / 2$ and $F\left(x_{i+1}\right)-F\left(b_{i}\right)=S$. Thus $S_{1}<S / 2$.

The mass of support that player $i$ gets after repositioning himself to $t$ is $S-$ $S_{1}+S_{2}>5 S / 4$, while the mass of support the candidates proposing $x_{i+1}$ get is $\left(2 S+S_{1}\right) / 2<5 S / 4$. Also, the mass of support the candidates proposing $x_{i-1}$ get is $<S$. Hence player $i$ wins the first round and increases his chances of winning the second round after repositioning himself to $t$.

Proof of Proposition 4. First we show that the equilibrium exists. Take any $2 \leq$ $r \leq n / 2$ and $e<\min \left\{d^{r}(F), e^{r}(F)\right\}$ and consider a strategy profile $s$ with a policy profile $\mathcal{X}_{s}=(\{m-e, m+e\}, \varrho)$ such that $\varrho\left(x_{1}\right)=\varrho\left(x_{2}\right)=r$. Suppose that $n>2 r$. Then there is no entry between $m-e$ and $m+e$ that would lead to at least tying for the first place in the first round, by the fact that $e<e^{r}(F)$. No entry at $m-e$ or $m+e$ is profitable as well, as in such a case the entrant cannot win the first round and, since $r \geq 2$, there will be at least two candidates proposing the other policy who will get to the second round. No entry outside the interval $[m-e, m+e]$ is profitable, because it always leads to losing in the second round. Consider any candidate under $s$. Exit by such a candidate is not profitable. Repositioning to the outside of the interval $[m-e, m+e]$ is not profitable, because it always leads to losing in the second round. Repositioning to the inside is not profitable by the fact that $e<d^{r}(F)$. Thus $s$ is a Nash equilibrium.

In the second part we show that if $s$ is a Nash equilibrium with policy profile $\mathcal{X}_{s}=\left(\left\{x_{1}, x_{2}\right\}, \varrho\right)$, then points $1-4$ have to be satisfied. For point 1 suppose that the opposite holds and assume that $x_{1}=m-e$, while $x_{2}=m+e^{\prime}$ with $e^{\prime}>e$ (the case $e^{\prime}<e$ is symmetric). Since, by Proposition 1, all the players must get the same support in the first round, so it must be that $\varrho\left(x_{1}\right)>\varrho\left(x_{2}\right)$. Moreover, by the same proposition, extreme policies must be at least binary, hence it follows that $\varrho\left(x_{1}\right) \geq 3$. On the other hand, by Proposition $2, \varrho\left(x_{1}\right)=2$ and we get a contradiction. Thus it must be that $e=e^{\prime}$.

For point 2 notice that $\varrho\left(x_{1}\right)=\varrho\left(x_{2}\right)$ follows immediately from point 1 . As noted above, it must also be that $\varrho\left(x_{1}\right) \geq 2$.

For point 3, assume the opposite. Suppose first that $r \geq 3$. In this case $e \geq d^{r}(F)$ implies that there is a position $t \in(m-e, m+e)$ such that a candidate proposing one of the extreme positions could deviate there and at least tie for the first place in the first round. Since such deviation guarantees winning the second round and does not decrease the chances of winning the first round, so it is always profitable. Thus if $s$ is a Nash equilibrium, then it must be that $e<d^{r}(F)$, if $r \geq 3$. Suppose now that $r=2$. In this case $e \geq d^{r}(F)$ implies that there is a position $t \in(m-e, m+e)$ such that a candidate proposing one of the extreme positions could deviate there and at least tie for the second place in the first round. If he wins or ties for the first place in the first round, then the deviation is profitable by 
the same arguments as those used above. Suppose then that he ties for the second place. Then it must be that the remaining candidate from the original position of the deviator wins the first round, and the deviator ties with the remaining two candidates. Moreover, getting to the second round guarantees him winning the elections. Hence the deviation increases his chances of winning and so it is profitable as well. Thus if $s$ is a Nash equilibrium, then it must be that $e<d^{r}(F)$, if $r=2$.

For point 4 notice that if $\varrho\left(\mathcal{X}_{s}\right)<n$ and $e \geq e^{r}(F)$, then one of the candidates standing out of the elections could always enter at some $t \in(m-e, m+e)$ and at least tie for the first place in the first round. Moreover, winning the first round guarantees him winning the elections. Hence entering would be always profitable for him, which would contradict the assumption that $s$ is a Nash equilibrium. Thus it must be that $e<e^{r}(F)$ if the number of candidates is smaller than the number of players.

Proof of Proposition 5. Suppose that $s$ is a Nash equilibrium with policy profile $\mathcal{X}_{s}=\left(\left\{x_{1}, x_{2}, x_{3}\right\}, \varrho\right)$.

We start with showing the following points, which give some general properties of any three-policy equilibria.

(i). $\varrho\left(x_{1}\right)=\varrho\left(x_{3}\right) \geq 2$ and $\varrho\left(x_{2}\right) \leq 2$.

(ii). If $\varrho\left(x_{2}\right)=2$, then $x_{2}=m$.

(iii). If $m-x_{1} \neq x_{3}-m$, then $\varrho\left(x_{2}\right)=2$ and $x_{1}=F^{-1}\left(\frac{1}{6}\right), x_{2}=m$ and $x_{3}=$ $F^{-1}\left(\frac{1}{6}\right)$

For point (i) notice first that, by Proposition $1, \varrho\left(x_{1}\right), \varrho\left(x_{3}\right) \geq 2$. Moreover, by point 3 of Proposition $2, \varrho\left(x_{2}\right) \leq 2$. Suppose now that $\varrho\left(x_{1}\right)>\varrho\left(x_{3}\right)$ (the case $\varrho\left(x_{1}\right)<\varrho\left(x_{3}\right)$ is symmetric). Then it must be that $\varrho\left(x_{1}\right) \geq 3$. Moreover, since $\varrho\left(x_{1}\right) \geq \varrho\left(x_{3}\right)+1$ and, by Proposition 1 , each candidate obtains the same mass of support, so $\left(x_{1}+x_{2}\right) / 2 \leq m$. Hence $m-x_{1}<x_{3}-m$, which contradicts point 1 of Proposition 2. Hence it must be that $\varrho\left(x_{1}\right)=\varrho\left(x_{3}\right)$.

For point (ii) assume that $\varrho\left(x_{2}\right)=2$. Then, by point 3 of Proposition 2 , the constituency of $x_{2}$ is balanced. Since $\varrho\left(x_{1}\right)=\varrho\left(x_{3}\right)$ and all the candidates obtain the same support in equilibrium, so the mass of voters to the left or $x_{2}$ must be equal to the mass of voters to the right of $x_{2}$, that is $x_{2}=m$.

For point (iii) we will use the following claim.

Claim 1. Suppose that $\varrho\left(x_{2}\right)=1$. Then the following points hold:

$$
\begin{aligned}
& \text { (i). if } m-x_{1} \leq x_{3}-m \text {, then } \sigma_{\mathrm{r}}\left(x_{1}\right) \leq \frac{\varrho\left(\mathcal{X}_{s}\right)+1}{2\left(\varrho\left(\mathcal{X}_{s}\right)-1\right)} \frac{1}{\varrho\left(\mathcal{X}_{s}\right)} \text {, and } \\
& \text { (ii). if } m-x_{1} \geq x_{3}-m \text {, then } \sigma_{1}\left(x_{k}\right) \leq \frac{\varrho\left(\mathcal{X}_{s}\right)+1}{2\left(\varrho\left(\mathcal{X}_{s}\right)-1\right)} \frac{1}{\varrho\left(\mathcal{X}_{s}\right)} \text {. }
\end{aligned}
$$

Proof. We will show point (i). Point (ii) can be shown by symmetric arguments. By Proposition 1, each candidate obtains the same mass of votes, $S=\frac{1}{\varrho\left(\mathcal{X}_{s}\right)}$. Suppose that $\varrho\left(x_{2}\right)=1$ and assume that $\sigma_{\mathrm{r}}\left(x_{1}\right)>T$, where $T=\frac{\varrho\left(\mathcal{X}_{s}\right)+1}{2\left(\varrho\left(\mathcal{X}_{s}\right)-1\right)} S$. Then there exists $x_{1}<z<b_{1}$ such that the mass of votes between $z$ and $b_{1}, F\left(b_{1}\right)-F(z)=T$. By moving to $x^{\prime}=2 z-x_{1}$ the candidate proposing $x_{2}$ gains the mass of votes between $z$ and $b_{1}, T$, and loses the mass of votes $U$ between $b_{2}$ and $b_{2}-b_{1}+z$, which is gained by the candidates proposing $x_{3}$. By point 2 of Proposition 2 it 


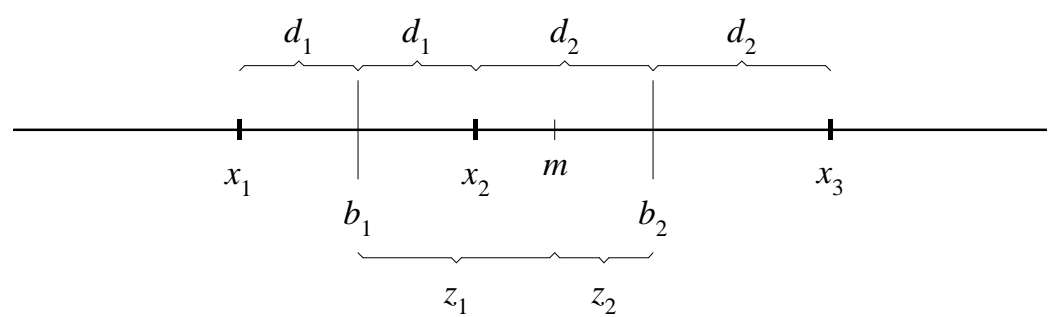

Figure 1: Equilibrium three-policy configuration with $m-x_{1}=x_{3}-m$.

must be that $b_{1}<m<b_{2}$. Moreover, $F(m)-F\left(b_{1}\right)=F\left(b_{2}\right)-F(m)=\frac{S}{2}$, as by point (i), $\varrho\left(x_{1}\right)=\varrho\left(x_{3}\right)$ and each candidate obtains the same mass of votes. Since $m-x_{1} \leq x_{3}-m$, so $b_{2}-b_{1}+z=\frac{x_{3}-x_{1}}{2}+z>m$ and so $U<\frac{S}{2}$. Now, the mass of votes gained by the candidate moving to $x^{\prime}$ is $T-U>\frac{S}{\varrho\left(\mathcal{X}_{s}\right)-1}$. On the other hand, the mass of votes gained by each candidate proposing $x_{3}$ is $\frac{2 U}{\varrho\left(\mathcal{X}_{s}\right)-1}<\frac{S}{\varrho\left(\mathcal{X}_{s}\right)-1}$. The candidates proposing $x_{1}$ only lose their support when the middle candidate moves to $x^{\prime}$. Notice also that $m-x^{\prime}<x_{3}-m$, as $m-x_{1} \leq x_{3}-m$. Hence the middle candidate wins the first round and always wins in the second round after repositioning. Thus there is a profitable deviation for him, which contradicts the assumption that $s$ is a Nash equilibrium.

Suppose now that $m-x_{1} \neq x_{2}-m$ and assume that $\varrho\left(x_{2}\right) \neq 2$. Then, by point (i), $\varrho\left(x_{2}\right)=1$. Suppose that $m-x_{1}<x_{2}-m$ (the other case is symmetric). Then, by point 1 of Proposition 2, $\sigma_{\mathrm{r}}\left(x_{1}\right)=\frac{1}{\varrho\left(\mathcal{X}_{s}\right)}>\frac{\varrho\left(\mathcal{X}_{s}\right)+1}{2\left(\varrho\left(\mathcal{X}_{s}\right)-1\right)} \frac{1}{\varrho\left(\mathcal{X}_{s}\right)}$ (recall that $\varrho\left(\mathcal{X}_{s}\right) \geq 5$ ). Thus we get a contradiction with Claim 1 . This shows that point (iii) holds.

For point 2 notice that point (iii) implies, in particular, that if $m-x_{1} \neq x_{2}-m$, then there are two binary policies with balanced support proposed in equilibrium. Hence, by Fact 1, such equilibria are non-generic and in any generic equilibrium it must hold that $m-x_{1} \neq x_{2}-m$. By point 2 of Proposition 2 it must be that $b_{1}<m<b_{2}$. Let $d_{1}=\frac{x_{1}+x_{2}}{2}, d_{2}=\frac{x_{2}+x_{3}}{2}, z_{1}=m-b_{1}$ and $z_{2}=b_{2}-m$ (c.f. Figure 1).

Then

$$
\begin{aligned}
& d_{1}+d_{2}=z_{1}+z_{2} \\
& d_{1}+z_{1}=d_{2}+z_{2} .
\end{aligned}
$$

Solving this system of equations we get $d_{1}=z_{2}$ and $d_{2}=z_{1}$. From the fact that $x_{1}=b_{1}-d_{1}, x_{2}=b_{2}+d_{1}$ and $x_{3}=b_{2}+d_{2}$ we obtain

$$
\begin{aligned}
& x_{1}=m+b_{1}-b_{2}, \\
& x_{2}=b_{1}+b_{2}-m, \\
& x_{3}=m-b_{1}+b_{2} .
\end{aligned}
$$

Since, by Proposition 1, all the candidates obtain the same mass of votes in the first round, so it must be that $b_{1}=q_{\frac{r}{2 r+1}}$ and $b_{2}=q_{\frac{r+1}{2 r+1}}$.

For point 3 notice that part of it is implied by point (i) and we only need to show that in any generic case $\varrho\left(x_{2}\right)=1$. Assume the opposite. Then, by point (i) 
it must be that $\varrho\left(x_{2}\right)=2$ and, by point (ii) it must be that $x_{2}=m$. This, together with point 2 implies that $q_{\frac{r}{2 r+1}}+q_{\frac{r+1}{2 r+1}}=2 m$, which is a non-generic property of the distribution. Thus in any generic case it must be that $\varrho\left(x_{2}\right)=1$.

Proof of Proposition 6. We start with the right to left implication and show that the conditions $1-4$ are necessary. Suppose that $s$ is a Nash equilibrium with the policy profile satisfying the properties stated in the proposition.

To see that condition 1 must hold assume the opposite. This means that there exists $t \in\left(0, m-q_{\frac{r}{2 r+1}}\right)$ such that

$$
F\left(q_{\frac{r+1}{2 r+1}}+t\right) \geq\left(\frac{r+1}{r}\right) F\left(q_{\frac{r}{2 r+1}}+t\right) .
$$

But then the candidate proposing $x_{2}$ could deviate to $x_{2}+t$ and obtain the mass of support

$$
S_{2}=F\left(q_{\frac{r+1}{2 r+1}}+t\right)-F\left(q_{\frac{r}{2 r+1}}+t\right) .
$$

The mass of support of any candidate proposing $x_{1}$ is then

$$
S_{1}=\frac{F\left(q_{\frac{r}{2 r+1}}+t\right)}{r}
$$

and, by (4), $S_{2} \geq S_{1}$. Moreover, $S_{1}>S_{3}$, the mass of support received by any candidate proposing $x_{3}$ after the deviation. Thus either the deviating candidate wins the first and the second round or he ties in the first round with the candidates proposing $x_{1}$ and always wins if selected for the second round. Hence the deviation is profitable, which contradicts the assumption that $s$ is a Nash equilibrium and so condition 1 is necessary. Analogous arguments can be used to show that condition 2 is necessary.

To see that condition 3 is necessary assume the opposite. Then there exists $t \in\left(m+q_{\frac{r}{2 r+1}}-q_{\frac{r+1}{2 r+1}}, q_{\frac{r}{2 r+1}}\right)$ such that the condition is violated. Thus a player could enter at $t+\frac{\frac{q}{2 r+1}-m}{2}$ obtaining a mass of votes $\geq \frac{1}{2 r+1}$, which allows him to win the first and the second round with positive probability. Since $n>2 r+1$, so there are player who are not candidates under $s$ and so there exists a profitable deviation for any such player. Condition 4 is necessary by similar arguments, as it prevents entry within the interval $\left(x_{2}, x_{3}\right)$.

Now we show that the conditions $1-4$ are sufficient. Suppose that $F$ satisfies the conditions and consider any strategy profile $s$ which yields the policy profile as described in the proposition and with

$$
x_{1}=m+q_{\frac{r}{2 r+1}}-q_{\frac{r+1}{2 r+1}}, x_{2}=q_{\frac{r}{2 r+1}}+q_{\frac{r+1}{2 r+1}}-m, x_{3}=m-q_{\frac{r}{2 r+1}}+q_{\frac{r+1}{2 r+1}} .
$$

Firstly, it is not profitable for any candidate to stay out of the elections, as each of them obtains the same mass of votes under $s$.

Secondly, there is no $z \in \mathbb{R}$ such that it would be profitable for the candidate proposing $x_{2}$ to deviate to $z$. Deviation to $z<x_{1}$ or $z>x_{3}$ is not profitable, because the deviating candidate would always loose in the second round. Deviation to $z=x_{1}$ or $z=x_{3}$ is not profitable, because no candidate proposing $z$ would win the first round then. Since $x_{1}$ and $x_{3}$ are symmetric about $m$, so two candidates 
proposing the other extreme would receive more votes and win. Deviation to $z \in$ $\left(x_{1}, x_{2}\right)$ is ruled out by condition 1 . For suppose that there is such a deviation which is profitable. This means that the deviating candidate does not loose with any of the candidates proposing $x_{3}$. Thus there must exist $t \in\left(0, x_{2}-b_{1}\right)$ such that

$$
F\left(x_{1}+t+x_{2}-b_{1}\right)-F\left(x_{1}+t\right) \geq \frac{F\left(x_{1}+t+x_{2}-b_{1}\right)}{r} .
$$

After inserting the values for $x_{1}, x_{2}$ and $b_{1}$ this simplifies to

$$
F\left(q_{\frac{r+1}{2 r+1}}+t\right) \geq\left(\frac{r+1}{r}\right) F\left(q_{\frac{r}{2 r+1}}+t\right),
$$

which contradicts condition 1 . Hence a deviation to $z \in\left(x_{1}, x_{2}\right)$ is not possible. By similar arguments it can be shown that a deviation to $z \in\left(x_{2}, x_{3}\right)$ is ruled out by condition 2 .

Thirdly, there is no profitable deviation to any $z \in \mathbb{R}$ by a candidate proposing the extreme position $x_{1}$. Deviation to $z<x_{1}$ or $z>x_{3}$ is not profitable as it never allows for winning in the second round. Deviation to the other extreme, $x_{3}$, or to $x_{2}$ leads to losing the first round. Suppose that there is a profitable deviation to some $z \in\left(x_{1}, x_{2}\right)$. This means that moving to $z$ the deviating candidate obtains the support $\geq \frac{1}{2 r+1}$, because to have a chance to proceed to the next round he cannot lose with any candidate proposing the extreme position $x_{3}$, the support of which is not affected by this deviation. This contradict condition 3 and so no such deviation is possible. Similarly, by condition 4 , there is no profitable deviation to $z \in\left(x_{2}, x_{3}\right)$.

Lastly, there is no profitable entry from any player who did not enter. Entries at positions outside the extreme positions are ruled out, because they do not allow for winning in the second round. Entries at positions $x_{1}, x_{2}$ and $x_{3}$ are ruled out, because they lead to losing the first round. Entries at positions within intervals $\left(x_{1}, x_{2}\right)$ and $\left(x_{2}, x_{3}\right)$ are ruled out by conditions 3 and 4 , respectively, by arguments given above.

Proof of Proposition 7. In the proof we will refer to the following values, uniquely determined by the distribution $F$, total number of contesting candidates $c \geq 1$, $1 \leq a \leq c$ and $1 \leq b \leq c$ :

$$
Q_{a, b}^{c}=\sum_{j=a}^{b}(-1)^{j-a+1} q_{\frac{j}{c}} .
$$

If $b-a$ is odd, then $Q_{a, b}^{c}$ is the sum of distances between subsequent even and odd $c$-quantiles, counted starting from the $a^{\prime}$ th one and up to the $b^{\prime}$ th one. If $b-a$ is even, then it is similar from the $a^{\prime}$ th to the $(b-1)^{\prime}$ th $c^{\prime}$ 'quantile, but additionally the $b$ 'th $c$-quantile is subtracted at the end.

We divide the proof into two lemmas that deal with cases of even and odd numbers of policies separately. In both cases we give the formulas specifying the policies proposed in any generic equilibrium, given the distribution $F$, the number of policies $k$ and the number of candidates $c$.

Lemma 2. Let $n$ be the number of players and suppose that $s$ is a Nash equilibrium with policy profile $\mathcal{X}_{s}=\left(\left\{x_{1}, \ldots, x_{k}\right\}, \varrho\right)$, where $\varrho\left(\mathcal{X}_{s}\right)=c$ and $k \geq 4$ is even. Then the following must hold in any generic case: 
1. $m-x_{1} \neq x_{k}-m$.

2. For all $1<i<k, \varrho\left(x_{i}\right)=1$.

3. $m-q_{\frac{1}{c}} \neq q_{\frac{c-1}{c}}-m$.

4. If $m-q_{\frac{1}{c}}<q_{\frac{c-1}{c}}-m$, then $\varrho\left(x_{1}\right)=2$ and $x_{i}=(-1)^{i-1}\left(q_{\frac{1}{c}}+2 Q_{2, i}^{c}\right)$, for $1 \leq i \leq k$.

5. If $m-q_{\frac{1}{c}}>q_{\frac{c-1}{c}}-m$, then $\varrho\left(x_{k}\right)=2$ and $x_{i}=(-1)^{i-1}\left(2\left(Q_{c-k, c-2}^{c}-\right.\right.$ $\left.\left.Q_{c-k, c-k+i-2}^{c}\right)-q_{\frac{c-1}{c}}\right)$, for $1 \leq i \leq k$.

Proof. For point 1 let $d_{i}=b_{i}-x_{i}$, for $1 \leq i \leq n-1$ and suppose that $m \in\left[b_{i-1}, b_{i}\right]$. By point 1 of Proposition $2,2 \leq i \leq n-1$. Let $z_{i-1}=m-b_{i-1}$ and let $z_{i}=b_{i}-m$. Then

$$
d_{i-1}+d_{i}=z_{i-1}+z_{i}
$$

Moreover, if $m-x_{1}=x_{k}-m$, then

$$
2 \sum_{j=1}^{i-2} d_{j}+d_{i-1}+z_{i-1}=z_{i}+d_{i}+2 \sum_{j=i+1}^{k-1} d_{j}
$$

Suppose that $i$ is even. From Equation (9) we get $d_{i-1}=z_{i-1}+z_{i}-d_{i}$. Inserting this in Equation (10) we obtain

$$
\sum_{j=1}^{i-2} d_{j}+z_{i-1}=\sum_{j=i}^{k-1} d_{j}
$$

From the fact that $d_{i}+d_{i+1}=b_{i+1}-b_{i}$, for $1 \leq i \leq k-1$, and from Equation (11) we get

$$
\sum_{j=1}^{\frac{i-2}{2}}\left(b_{2 j}-b_{2 j-1}\right)+m-b_{i-1}=\sum_{j=\frac{i}{2}}^{\frac{k-2}{2}}\left(b_{2 j+1}-b_{2 j}\right) .
$$

By Proposition 1 the policy profile $\mathcal{X}_{s}$ must be even and, as we observed in Section 3.1 , each of $b_{i}$ 's is a proper $\varrho\left(\mathcal{X}_{s}\right)$-quantile of the distribution $F$ (c.f. Equation (1)). Thus for any fixed policy profile, Equation (12) is a non-generic property of a distribution. Hence in any generic case with $i$ being even it must be that $m-x_{1} \neq x_{k}-m$.

Suppose now that $i$ is odd. The analysis here is analogous to the previous case. From Equation (9) we get $d_{i}=z_{i-1}+z_{i}-d_{i-1}$. Inserting this in Equation (10) we obtain

$$
\sum_{j=1}^{i-1} d_{j}=z_{i}+\sum_{j=i+1}^{k-1} d_{j}
$$

From the fact that $d_{i}+d_{i+1}=b_{i+1}-b_{i}$, for $1 \leq i \leq k-1$, and from Equation (13) we get

$$
\sum_{j=1}^{\frac{i-1}{2}}\left(b_{2 j}-b_{2 j-1}\right)=b_{i}-m+\sum_{j=\frac{i+1}{2}}^{\frac{k-2}{2}}\left(b_{2 j+1}-b_{2 j}\right) .
$$


Again, this equation is a non-generic property of a distribution, hence in any generic case with $i$ being odd it must be that $m-x_{1} \neq x_{k}-m$.

Point 2 follows immediately from point 1 , Fact 1 , and points 1 and 3 of Proposition 2 .

For points 4 and 5 notice that since $m-x_{1} \neq x_{k}-m$ so, by point 1 of Proposition 2 , either $\varrho x_{1}=2$, its constituency is balanced and $m-x_{1}<x_{k}-m$, or $\varrho x_{k}=2$, its constituency is balanced and $m-x_{1}>x_{k}-m$. This implies, in particular, that either $x_{1}=q_{\frac{1}{c}}$ or $x_{k}=q_{\frac{c-1}{c}}$, respectively. Moreover, if $m-q_{\frac{1}{c}}<q_{\frac{c-1}{c}}-m$, then it must be that $\varrho x_{1}=2$ and $x_{1}=q_{\frac{1}{c}}$. Similarly, if $m-q_{\frac{1}{c}}>q_{\frac{c-1}{c}}-m$, then it must be that $\varrho x_{k}=2$ and $x_{k}=q_{\frac{c-1}{c}}$. Suppose that $x_{1}=q_{\frac{1}{c}}$. Since each of the internal policies is proposed by exactly one candidate and each candidate obtains the same support, so $x_{i}$ must be the reflection of $x_{i-1}$ in $q_{\frac{i}{c}}$. It can be easily checked that $x_{i}=(-1)^{i-1}\left(q_{\frac{1}{n}}+2 Q_{2, i-1}^{c}\right)$, for $1 \leq i \leq k$, which shows that points 4 holds. Point 5 can be shown analogously.

Lemma 3. Let $n$ be the number of players and suppose that $s$ is a Nash equilibrium with policy profile $\mathcal{X}_{s}=\left(\left\{x_{1}, \ldots, x_{k}\right\}, \varrho\right)$, where $\varrho\left(\mathcal{X}_{s}\right)=c$ and $k \geq 5$ is odd. Then the following must hold in any generic case:

1. For all $1<i<k, \varrho\left(x_{i}\right)=1$.

2. If $2 m \leq q_{\frac{1}{c}}+q_{\frac{k}{c}}$, or $q_{\frac{1}{c}}+q_{\frac{k}{c}}<2 m<q_{\frac{2}{c}}+q_{\frac{k+1}{c}}$ and $m-q_{\frac{1}{c}}<Q_{2, k}^{c}$, then $\varrho\left(x_{1}\right)=2$ and $x_{i}=(-1)^{i-1}\left(q_{\frac{1}{c}}+2 Q_{2, i}^{c}\right)$, for all $1 \leq i \leq k$.

3. If $q_{\frac{1}{c}}+q_{\frac{k}{c}}<2 m<q_{\frac{2}{c}}+q_{\frac{k+1}{c}}$ and $m-q_{\frac{1}{c}} \geq Q_{2, k}^{c}$, then $\varrho\left(x_{1}\right)=2$ and $x_{i}=(-1)^{i-1}\left(m+2 Q_{2, i}^{c}-Q_{2, k}^{c}\right)$, for all $1 \leq i \leq k$.

4. If $q_{\frac{r-1}{c}}+q_{\frac{k+r-2}{c}}<2 m<q_{\frac{r}{c}}+q_{\frac{k+r-1}{c}}$ with $3 \leq r \leq c-k-1$, then $\varrho\left(x_{1}\right)=r$ and ${ }^{c} x_{i}=(-1)^{i-1}\left(m+2 Q_{r, r+i-2}^{c}-Q_{r, r+k-2}^{c}\right)$, for all $1 \leq i \leq k$.

5. If $q_{\frac{c-k-1}{c}}+q_{\frac{c-2}{c}}<2 m<q_{\frac{c-k}{c}}+q_{\frac{c-1}{c}}$ and $q_{\frac{c-1}{c}}-m \geq Q_{c-k, c-2}^{c}$, then $\varrho\left(x_{1}\right) \stackrel{c}{=} c-k^{c}$ and $x_{i}=(-1)^{i-1}\left(m+2 Q_{c-k, c-k+i-2}^{c}-Q_{c-k, c-2}^{c}\right)$, for all $1 \leq i \leq k$.

6. If $q_{\frac{c-k}{c}}+q_{\frac{c-1}{c}} \leq 2 m$, or $q_{\frac{c-k-1}{c}}+q_{\frac{c-2}{c}}<2 m<q_{\frac{c-k}{c}}+q_{\frac{c-1}{c}}$ and $q_{\frac{c-1}{c}}-$ $m<{ }^{c} Q_{c-k, c-2}^{c}$, then $\varrho\left(x_{1}\right)={ }^{c}-k$ and $x_{i}=(-1)^{i-1}\left(q_{\frac{c-1}{c}}-2\left(Q_{c-k, c-2}^{c}-\right.\right.$ $\left.Q_{c-k, c-k+i-2}^{c}\right)$, for all $1 \leq i \leq k$.

Proof. We start with showing that positions of the policies are uniquely determined by $\varrho$ and the symmetry of extreme policies with respect to $m$. Suppose first that $m-x_{1}=x_{k}-m$. Like in proof of Lemma 2 , let $d_{i}=b_{i}-x_{i}$, for $1 \leq i \leq n-1$ and suppose that $m \in\left[b_{i-1}, b_{i}\right]$. By point 2 of Proposition $2,2 \leq i \leq n-1$. Let $z_{i-1}=m-b_{i-1}$ and let $z_{i}=b_{i}-m$. Then

$$
d_{i-1}+d_{i}=z_{i-1}+z_{i}
$$

Moreover, if $m-x_{1}=x_{k}-m$, then

$$
2 \sum_{j=1}^{i-2} d_{j}+d_{i-1}+z_{i-1}=z_{i}+d_{i}+2 \sum_{j=i+1}^{k-1} d_{j} .
$$


Suppose that $i$ is even. Like in proof of Lemma 2 we get

$$
\sum_{j=1}^{i-2} d_{j}+z_{i-1}=\sum_{j=i}^{k-1} d_{j}
$$

From this and from the fact that $d_{i}+d_{i+1}=b_{i+1}-b_{i}$, for $1 \leq i \leq k-1$, we get

$$
\sum_{j=1}^{\frac{i-2}{2}}\left(b_{2 j}-b_{2 j-1}\right)+m-b_{i-1}=d_{i}+\sum_{j=\frac{i+2}{2}}^{\frac{k-1}{2}}\left(b_{2 j}-b_{2 j-1}\right) \text {. }
$$

From this we obtain

$$
\sum_{j=1}^{i-1}(-1)^{j} b_{j}+m+b_{i}=d_{i}+\sum_{j=i}^{k-1}(-1)^{j} b_{j}
$$

and further

$$
d_{i}=b_{i}+m+2 \sum_{j=1}^{i-1}(-1)^{j} b_{j}-\sum_{j=1}^{k-1}(-1)^{j} b_{j} .
$$

By similar analysis for $i$ being odd we get

$$
d_{i}=b_{i}-\left(m+2 \sum_{j=1}^{i-1}(-1)^{j} b_{j}-\sum_{j=1}^{k-1}(-1)^{j} b_{j}\right) .
$$

Hence

$$
d_{i}=b_{i}+(-1)^{i}\left(m+2 \sum_{j=1}^{i-1}(-1)^{j} b_{j}-\sum_{j=1}^{k-1}(-1)^{j} b_{j}\right) .
$$

From Equation (22) we get

$$
d_{1}=b_{1}-m+\sum_{j=1}^{k-1}(-1)^{j} b_{j}
$$

Since $x_{1}=b_{1}-d_{1}$, so

$$
x_{1}=m-\sum_{j=1}^{k-1}(-1)^{j} b_{j} .
$$

From the fact that $x_{i}=2 b_{i-1}-x_{i-1}$, for $2 \leq i \leq k-1$, we get

$$
\begin{aligned}
x_{i} & =(-1)^{i-1}\left(x_{1}+2 \sum_{j=1}^{i-1}(-1)^{j} b_{j}\right) \\
& =(-1)^{i-1}\left(m+2 \sum_{j=1}^{i-1}(-1)^{j} b_{j}-\sum_{j=1}^{k-1}(-1)^{j} b_{j}\right) .
\end{aligned}
$$

By Proposition 1 the policy profile $\mathcal{X}_{s}$ must be even and, as we observed in Section 3.1 , each of $b_{i}$ 's is a proper $\varrho\left(\mathcal{X}_{s}\right)$-quantile of the distribution $F$ (c.f. Equation $(1))$. Hence all $x_{i}$, with $1 \leq x_{i} \leq k$, are uniquely determined by $\varrho$ in this case. 
If $m-x_{1}<x_{k}-m$, then, by point 1 of Proposition 2 , it must be that $\varrho\left(x_{1}\right)=2$ and its constituency is balanced. Hence $x_{1}=q_{\frac{1}{c}}$ and all $x_{i}$, for the remaining $2 \leq i \leq k$, can be determined by the fact that the policy profile is even and $x_{i+1}$ is the reflection of $x_{i}$ in $b_{i}$. Similarly, the policies $x_{i}$ are uniquely determined if $m-x_{1}>x_{k}-m$.

For point 1 suppose that there is an internal policy $x_{i}$ which is not proposed by one candidate. By point 3 of Proposition 2 it must be proposed by two candidates and the constituency of $x_{i}$ must be balanced. Moreover, by Fact 1, in any generic case there can be at most one such policy. Since each candidate obtains the same mass of votes in the first round, so

$$
x_{i}=q_{\frac{\varrho\left(x_{1}\right)+i-1}{c}} .
$$

On the other hand, from Equation (25) and from the fact that all the internal positions to left of $x_{i}$ are unary and all candidates are tied, we have

$$
\begin{aligned}
x_{i} & =(-1)^{i-1}\left(m+2 \sum_{j=\varrho\left(x_{1}\right)}^{\varrho\left(x_{1}\right)+i-2}(-1)^{j-\varrho\left(x_{1}\right)+1} q_{\frac{j}{c}}-\sum_{j=\varrho\left(x_{1}\right)}^{\varrho\left(x_{1}\right)+k-2}(-1)^{j-\varrho\left(x_{1}\right)+1} q_{\frac{j}{c}}\right) \\
& =(-1)^{i-1}\left(m+2 Q_{\varrho\left(x_{1}\right), \varrho\left(x_{1}\right)+i-2}^{c}-Q_{\varrho\left(x_{1}\right), \varrho\left(x_{1}\right)+k-2}^{c}\right) .
\end{aligned}
$$

Both Equations (26) and (27) being satisfied is a non-generic property of the distribution. Hence in any generic case each internal policy must be proposed by exactly one candidate.

For points $2-6$ notice first that by point 1 of Proposition 2 and by the fact that all the candidates obtain the same support in the first round and since all the internal policies must be unary in any generic case, so in any generic case it must be that

$$
\begin{gathered}
q_{\underline{\underline{\rho\left(x_{1}\right)-1}}} \leq x_{1}<q_{\underline{\underline{\varrho\left(x_{1}\right)}}} \text { and } \\
q_{\underline{\underline{\rho\left(x_{1}\right)+k-2}}} \leq x_{k} \leq q_{\underline{\underline{\rho\left(x_{1}\right)+k-1}}}^{c}
\end{gathered}
$$

Suppose that $m-x_{1}=x_{k}-m$. This, together with Inequalities $(28-29)$ implies that

$$
\begin{array}{r}
m-q_{\underline{\underline{\rho\left(x_{1}\right)-1}}}>q_{\underline{\underline{Q\left(x_{1}\right)+k-2}}}^{c}-m \text { and } \\
m-q_{\frac{\rho\left(x_{1}\right)}{c}}<q_{\frac{\rho\left(x_{1}\right)+k-1}{c}}-m .
\end{array}
$$

Suppose that $m-x_{1}<x_{k}-m$. By point 1 of Proposition 2 it must be that $\varrho\left(x_{1}\right)=2$ and, by Inequalities $(28-29)$, it must be that

$$
m-q_{\frac{1}{c}}<q_{\frac{k+1}{c}}-m
$$

Similarly, if $m-x_{1}>x_{k}-m$, then it must be that $\varrho\left(x_{k}\right)=2\left(\right.$ and $\left.\varrho\left(x_{1}\right)=c-k\right)$ and, by Inequalities $(28-29)$, it must be that

$$
m-q_{\frac{c-k-1}{c}}>q_{\frac{c-1}{c}}-m .
$$

Suppose now that $q_{\frac{r}{c}}+q_{\frac{r+k-1}{c}}<2 m<q_{\frac{r+k-2}{c}}+q_{\frac{r-1}{c}}$ with $3 \leq r \leq c-k-1$. Then, by Inequalities $(28-33)$, it must be that $\varrho\left(x_{1}\right) \stackrel{c}{=} r$ and, by Proposition 1 , 
it must be that $m-x_{1}=x_{k}-m$. Each $x_{i}$, with $1 \leq i \leq k$ must be like in Equation (27).

Suppose that $2 m \leq q_{\frac{r}{c}}+q_{\frac{r+k-1}{c}}$. Then, by Inequalities $(28-33)$, it must be that $\varrho\left(x_{1}\right)=2, m-x_{1}<x_{k}-m$ and the constituency of $x_{1}$ is balanced. Since the mass of support obtained by each candidate in the first round must be the same and in any generic case internal policies are unary, so it can be easily shown that

$$
x_{i}=(-1)^{i-1}\left(q_{\frac{1}{c}}+2 Q_{2, i}^{c}\right),
$$

for $1 \leq i \leq k$. The case of $q_{\frac{r+k-2}{c}}+q_{\frac{r-1}{c}} \leq 2 m$ can be shown analogously.

Lastly suppose that $q_{\frac{2}{c}}+q_{\frac{k+1}{c}}<2 m<q_{\frac{k}{c}}+q_{\frac{1}{c}}$. Then, by Inequalities (28 - 33), it must be that $\varrho\left(x_{1}\right)=2$ and either $m-x_{1}=x_{k}-m$ or $m-x_{1}<x_{k}-m$. If $m-x_{1}=x_{k}-m$, then, by Equation (27), $x_{1}=m-Q_{2, k}^{c}$. Since, by point 1 of Proposition 2, it must be that $x_{1} \geq q_{\frac{1}{c}}$, so if $m-q_{\frac{1}{c}}<Q_{2, k}^{c}$, then it must be that $m-x_{1}<x_{k}-m$ and $x_{1}=q_{\frac{1}{c}}$. The policies proposed must be like in Equation (34). If $m-x_{1}<x_{k}-m$, then, by Equation (34), $x_{k}=q_{\frac{1}{c}}+2 Q_{2, k}^{c}$. By the facts that $m-x_{1}<x_{k}-m$ and $x_{1}=q_{\underline{1}}$, it must be that $m-q_{\underline{1}}{ }^{c}<Q_{2, k}^{c}$. Thus if $m-q_{\frac{1}{c}} \geq Q_{2, k}^{c}$, then it must be that $m-x_{1}=x_{k}-m$ and. The policies proposed must be like in Equation (27). The case of $q_{\frac{c-k}{c}}+q_{\frac{c-1}{c}}<2 m<q_{\frac{c-2}{c}}+q_{\frac{c-k-1}{c}}$ can be shown analogously. tion.

Both lemmas shown above imply immediately the result stated in the proposi-

\section{References}

Blais, A., Massicotte, L., Dobrzynska, A., 1997. Direct presidential elections: a world summary. Electoral Studies 16 (4), 441-455.

Bordignon, M., Tabellini, G., 2009. Moderating political extremism: Single round vs runoff elections under plurality rule. Working Papers 348, IGIER (Innocenzo Gasparini Institute for Economic Research), Bocconi University.

Callander, S., 2005. Duverger's hypothesis, the run-off rule, and electoral competition. Political Analysis 13, 209-232.

Cox, G. W., 1997. Making votes count : strategic coordination in the world's electoral system. Political economy of institutions and decisions. Cambridge University Press, New York.

Downs, A., 1957. An Economic Theory Of Democracy. New York: Harper and Row.

Dziubiński, M., Roy, J., 2009. Hotelling-Downs competition with free entry when voters have an option to contest, unpublished. 
Grofman, B., 2008. A taxonomy of runoff methods. Electoral Studies 27 (3), 395-399.

Haan, M., Volkerink, B., 2001. A runoff system restores the principle of minimum differentiation. European Journal of Political Economy 17 (1), $157-162$.

Hotelling, H., 1929. Stability in competition. Economic Journal 39 (153), $41-57$.

Matsushima, N., 2007. Uncertainty of voters' preferences and differentiation in a runoff system. European Journal of Political Economy 23 (4), 11851189 .

Messner, M., Polborn, M., 2007. Strong and coalition-proof political equilibria under plurality and runoff rule. International Journal of Game Theory 35 (2), 287-314.

Osborne, M. J., 1993. Candidate positioning and entry in a political competition. Games and Economic Behavior 5 (1), 133-151.

Osborne, M. J., Slivinski, A., 1996. A model of political competition with citizen-candidates. Quarterly Journal of Economics 111 (1), 65-96.

Palfrey, T. R., 1984. Spatial equilibrium with entry. Review of Economic Studies 51, 139-156.

Wright, S. G., Riker, W. H., 1989. Plurality and runoff systems and numbers of candidates. Public Choice 60 (2), 155-175. 